\title{
Topological insulators with inversion symmetry
}

\author{
Liang Fu and C. L. Kane \\ Department of Physics and Astronomy, University of Pennsylvania, Philadelphia, Pennsylvania 19104, USA
}

(Received 14 November 2006; revised manuscript received 17 January 2007; published 2 July 2007)

\begin{abstract}
Topological insulators are materials with a bulk excitation gap generated by the spin-orbit interaction that are different from conventional insulators. This distinction is characterized by $Z_{2}$ topological invariants, which characterize the ground state. In two dimensions, there is a single $Z_{2}$ invariant that distinguishes the ordinary insulator from the quantum spin-Hall phase. In three dimensions, there are four $Z_{2}$ invariants that distinguish the ordinary insulator from "weak" and "strong" topological insulators. These phases are characterized by the presence of gapless surface (or edge) states. In the two-dimensional quantum spin-Hall phase and the threedimensional strong topological insulator, these states are robust and are insensitive to weak disorder and interactions. In this paper, we show that the presence of inversion symmetry greatly simplifies the problem of evaluating the $Z_{2}$ invariants. We show that the invariants can be determined from the knowledge of the parity of the occupied Bloch wave functions at the time-reversal invariant points in the Brillouin zone. Using this approach, we predict a number of specific materials that are strong topological insulators, including the semiconducting alloy $\mathrm{Bi}_{1-x} \mathrm{Sb}_{x}$ as well as $\alpha$-Sn and $\mathrm{HgTe}$ under uniaxial strain. This paper also includes an expanded discussion of our formulation of the topological insulators in both two and three dimensions, as well as implications for experiments.
\end{abstract}

DOI: 10.1103/PhysRevB.76.045302

PACS number(s): 73.43. $-\mathrm{f}, 72.25 . \mathrm{Hg}, 73.20 .-\mathrm{r}, 85.75 .-\mathrm{d}$

\section{INTRODUCTION}

In elementary solid-state physics textbooks, an insulator is described as a material with an energy gap separating filled and empty energy bands. A more sophisticated definition of an insulator is that of a material for which all electronic phenomena are local. ${ }^{1}$ Such a material is insensitive to boundary conditions, so that in a multiply connected sample, such as a ring, there is exponentially small sensitivity to magnetic flux threading the holes. The simplest atomic insulator, in which all electrons are tightly bound to atoms in closed shells, clearly satisfies both properties. Ionic and covalent insulators do too. These band insulators are topologically equivalent in the sense that the Hamiltonian can be adiabatically transformed into an atomic insulator without going through any phase transitions. With regards to their low-energy electronic behavior, conventional insulators are equivalent to atomic insulators.

The existence of a bulk energy gap does not guarantee the insensitivity to boundary conditions, and there exist phases with bulk gaps, which are topologically distinct. In addition to exotic strongly correlated phases, ${ }^{2,3}$ this fact arises even for noninteracting electrons described within band theory. The simplest example is the integer quantum Hall effect (IQHE). In two dimensions, a magnetic field introduces a cyclotron gap between Landau levels, which may be viewed as energy bands in the magnetic Brillouin zone. This phase can exist even without Landau levels in the absence of a uniform magnetic field, ${ }^{4}$ though a necessary condition is that time-reversal symmetry be broken. Based on the band structure alone, it is difficult to tell the difference between the IQHE state and a band insulator. The distinction between the two is a topological property of the occupied bands which is encoded into the Chern integer introduced by Thouless et al. ${ }^{5}$ Three-dimensional generalizations of the IQHE state, which may be viewed as layered two-dimensional (2D) states, are indexed by a triad of Chern integers. ${ }^{6}$ A hallmark of the
IQHE phases, which is intimately related to their topology, is the existence of gapless chiral edge states which are robust in the presence of disorder. ${ }^{7,8}$

Recently, new topological insulating phases for systems with time-reversal symmetry have been discovered. ${ }^{9-15}$ In two dimensions, the quantum spin-Hall phase is distinguished from a band insulator by a single $Z_{2}$ invariant. ${ }^{10}$ This phase exhibits gapless spin-filtered edge states, which allow for dissipationless transport of charge and spin at zero temperature and are protected from weak disorder and interactions by time-reversal symmetry. In three dimensions, a timereversal invariant band structure is characterized by four $Z_{2}$ invariants. ${ }^{13-15}$ Three of the invariants rely on the translational symmetry of the lattice and are not robust in the presence of disorder, leading to "weak topological insulators." The fourth invariant, however, is robust and distinguishes the "strong topological insulator" (STI).

Nontrivial $Z_{2}$ invariants imply the existence of gapless surface states. In particular, in the STI phase, the surface states form a two-dimensional "topological metal," in which the Fermi arc encloses an odd number of Dirac points. ${ }^{15}$ This leads to a quantized Berry's phase of $\pi$ acquired by an electron circling the surface Fermi arc, which does not change under continuous perturbations. ${ }^{16,17}$ The $\pi$ Berry's phase also signifies that with disorder, the surface states are in the symplectic universality class and exhibit antilocalization. ${ }^{18}$ Thus, the metallic surface states form a unique phase, which cannot be realized in a conventional two-dimensional electron system for which Dirac points must come in pairs. ${ }^{19}$

The purpose of this paper is twofold. First, we will explain the formulation of the $Z_{2}$ invariants in somewhat more detail than in Ref. 15, emphasizing the physical meaning of the invariants and their relation to the surface states. Second, we will develop a method for evaluating the $Z_{2}$ invariants in systems which have inversion symmetry. This allows us to predict a number of specific materials to be strong topological insulators. 
Most insulators are conventional insulators. In order to find topological insulators experimentally, it is necessary to develop criteria for recognizing them from their bulk band structure. Clearly, a necessary condition is the existence of a bulk energy gap which owes its existence to the spin-orbit interaction. However, evaluating the $Z_{2}$ invariants for a given band structure is, in general, a difficult problem. To date, three general approaches have been used.

(1) One powerful approach is to exploit adiabatic continuity to a Hamiltonian which has extra symmetry. We used this method to identify the quantum spin-Hall phase in graphene $^{9,10}$ by arguing that the Hamiltonian for graphene is adiabatically connected to a Hamiltonian in which the spin $S_{z}$ is conserved. With this extra conservation law, the system can be characterized by a spin Chern number, which describes the quantized spin-Hall conductivity. ${ }^{20,21}$ The $Z_{2}$ invariant can then be identified with the parity of the spin Chern number. In the presence of $S_{z}$ nonconserving terms (which are inevitably present), the spin Chern number loses its meaning. ${ }^{22}$ However, the $Z_{2}$ invariant retains its value and characterizes the quantum spin-Hall phase.

Adiabatic continuity can also be used to establish that a material is a band insulator if an adiabatic path can be found which connects the material to an "atomic" limit. Moreover, it can be argued that the $Z_{2}$ invariant changes at an appropriate quantum phase transition, where the bulk energy gap goes to zero. ${ }^{12,14}$ In general, this approach requires a continuous path to be found which connects the Hamiltonian in question to a known phase.

(2) It is also possible to evaluate the $Z_{2}$ invariant directly with the knowledge of the Bloch wave functions for the occupied energy bands. In Ref. 22, we established a formula for the invariant in terms of a Brillouin-zone integral. This is analogous to the calculation of the Chern number as an integral of the gauge invariant Berry's curvature. 5,23 However, unlike the Chern invariant, the integral for the $Z_{2}$ invariant also involves the Berry's potential and requires a gauge in which the wave functions are globally continuous. Since time-reversal symmetry requires the Chern invariant to vanish, a globally continuous gauge is guaranteed to exist. However, finding a continuous gauge is not always simple.

(3) A third approach is to characterize the zeros of Pfaffian function introduced Ref. 10. Though the Pfaffian is not gauge invariant, its zeros can be determined without specifying a continuous gauge. While this approach is tedious (especially in three dimensions), it has been successfully implemented by Murakami ${ }^{24}$ to show that two-dimensional bismuth bilayers realize a quantum spin-Hall phase.

In this paper, we will show that the presence of inversion symmetry greatly simplifies the problem of identifying the $Z_{2}$ invariants. We show that the invariants can be determined from the knowledge of the parity of the occupied band eigenstates at the eight (or four in two dimensions) time-reversal invariant momenta $\Gamma_{i}$ in the Brillouin zone. Specifically, we will show that the $Z_{2}$ invariants are determined by the quantities

$$
\delta_{i}=\prod_{m=1}^{N} \xi_{2 m}\left(\Gamma_{i}\right) .
$$

Here, $\xi_{2 m}\left(\Gamma_{i}\right)= \pm 1$ is the parity eigenvalue of the $2 m$ th occupied energy band at $\Gamma_{i}$, which shares the same eigenvalue $\xi_{2 m}=\xi_{2 m-1}$ with its Kramers degenerate partner. The product involves the $2 \mathrm{~N}$ occupied bands. The $Z_{2}$ invariant $\nu=0,1$, which distinguishes the quantum spin-Hall phase in two dimensions and the strong topological insulator in three dimensions, is then given by the product of all the $\delta_{i}$ 's,

$$
(-1)^{\nu}=\prod_{i} \delta_{i}
$$

The other three "weak" topological invariants in three dimensions are also determined by $\delta_{i}$. Since the parity eigenvalues $\xi_{n}\left(\Gamma_{i}\right)$ are tabulated in the band theory literature, this allows us to identify inversion symmetric topological insulating materials. Moreover, exploiting adiabatic continuity allows us to identify topological insulators which do not have inversion symmetry but are adiabatically connected to materials which have inversion symmetry.

Applying the above approach, we predict that the following narrow gap semiconductors are strong topological insulators: (1) the alloy $\mathrm{Bi}_{1-x} \mathrm{Sb}_{x}$, which is semiconducting for $0.07<x<0.22$, (2) $\alpha$-Sn and HgTe under uniaxial strain, and (3) the alloy $\mathrm{Pb}_{1-x} \mathrm{Sn}_{x} \mathrm{Te}$ under uniaxial strain for $x \sim x_{c}$ in the vicinity of the band inversion transition. The materials (2) and (3) were suggested by Murakami et al..$^{25}$ as candidates for spin-Hall insulators. Those authors argued that those materials share a large spin-Hall conductivity, as calculated by a Kubo formula. Our analysis of these materials is rather different, and we will show that PbTe is a conventional insulator, despite its large spin-Hall conductivity, while strained $\alpha$-Sn and HgTe are topological insulators.

In Sec. II, we will present an expanded discussion of our formulation of the $Z_{2}$ invariants. Then, in Sec. III, we will derive Eqs. (1.1) and (1.2) for problems with inversion symmetry. In Sec. IV, we will apply our method to a class of four band tight-binding models, which includes the graphene model as well as the three-dimensional (3D) model introduced in Ref. 15. In Sec. V, we will apply Eqs. (1.1) and (1.2) to deduce the $Z_{2}$ invariants of several real materials based on their known band structures. Readers uninterested in the technical details can skip directly to Sec. V to read about these applications. Finally, in Sec. VI, we will conclude with a brief discussion of the experimental implications for the topological insulating phases.

\section{II. $Z_{2}$ INVARIANTS IN TWO AND THREE DIMENSIONS}

In this section, we will review our formulation of the topological insulating phases. We begin in Sec. II A by defining the time-reversal polarization. In Sec. II B, we develop the $Z_{2}$ characterization of a band structure as a topological property of the occupied Bloch wave functions. In Sec. II C, we show how the $Z_{2}$ invariants determine the surface-state spectrum. In Sec. II C, we consider a more general formulation of the $Z_{2}$ invariant as a sensitivity of a bulk crystal to boundary conditions. 


\section{A. Time-reversal polarization}

In Ref. 22, we introduced the concept of the time-reversal polarization, in the same spirit as charge polarization. This allows for an interpretation of the $Z_{2}$ invariants using a Laughlin-type gedanken experiment on a cylinder. ${ }^{26}$ In the ordinary quantum Hall effect, the Chern invariant can be interpreted in a similar way. To motivate the time-reversal polarization, we therefore begin with a discussion of the charge polarization.

The charge polarization determines the surface charges present in a finite system. Since electrons may be added or removed from a surface, the charge polarization is defined only modulo an integer. ${ }^{27-30}$ However, changes in the charge polarization induced by adiabatic changes in the Hamiltonian are well defined. In Laughlin's gedanken experiment for the integer quantum Hall effect, a quantum of magnetic flux $h / e$ is adiabatically inserted in a cylindrical quantum Hall sample at filling $\nu=N$. The resulting transfer of $N$ electrons from one end of the cylinder to the other can be interpreted as a change in the charge polarization of the cylinder. The Chern invariant, which characterizes the integer quantum Hall state, precisely characterizes this quantized change in charge polarization.

The time-reversal polarization is a $Z_{2}$ quantity, which characterizes the presence or absence of a Kramers degeneracy associated with a surface. Like the charge polarization, its value can be changed by adding an extra electron to the surface. Thus, by itself, the time-reversal polarization is not meaningful. However, changes in the time-reversal polarization due to adiabatic changes in the bulk Hamiltonian are well defined. Specifically, the change in the time-reversal polarization when half a flux quantum $h / 2 e$ is threaded through a cylinder defines a $Z_{2}$ invariant, which is analogous to the Chern invariant, and distinguishes topological insulators.

\section{B. $Z_{2}$ invariants characterizing a band structure}

The topological invariant characterizing a twodimensional band structure may be constructed by imagining a long cylinder whose axis is parallel to a reciprocal-lattice vector $\mathbf{G}$ and which has a circumference of a single lattice constant. Then, the magnetic flux threading the cylinder plays the role of the circumferential (or "edge") crystal momentum $k_{x}$, with $\Phi=0$ and $\Phi=h / 2 e$ corresponding to two edge time-reversal invariant momenta $k_{x}=\Lambda_{1}$ and $k_{x}=\Lambda_{2}$. The $Z_{2}$ invariant characterizes the change in the Kramers degeneracy at the ends of this one-dimensional system between $k_{x}=\Lambda_{1}$ and $k_{x}=\Lambda_{2}$.

For a three-dimensional crystal, imagine a "generalized cylinder" which is long in one direction (parallel to $\mathbf{G}$ ) but, in the other two directions, has a width of one lattice constant with periodic boundary conditions. While this structure cannot be pictured as easily as a regular cylinder, a distorted (but topologically equivalent) version can be visualized as a torus with a finite thickness. This "Corbino donut" is analogous to the generalized cylinder in the same way the Corbino disk is analogous to the regular cylinder. The "long" direction corresponds to the thickness of the torus, and the two ends correspond to the inner and outer surfaces. This system can be threaded by two independent magnetic fluxes, and they correspond to the two components of the momentum perpendicular to $\mathbf{G}$. There are four time-reversal invariant surface momenta $\Lambda_{a}$, corresponding to the two fluxes being either 0 or $h / 2 e$. The band structure can be characterized by the difference in the time-reversal polarization between any pair.

The $Z_{2}$ invariants can be deduced from the topological structure of the Bloch wave functions of the bulk crystal in the Brillouin zone. Consider a time-reversal invariant periodic Hamiltonian $\mathcal{H}$ with $2 \mathrm{~N}$ occupied bands characterized by Bloch wave functions

$$
\left|\psi_{n, \mathbf{k}}\right\rangle=e^{i \mathbf{k} \cdot \mathbf{r}}\left|u_{n, \mathbf{k}}\right\rangle
$$

Here, $\left|u_{n, \mathbf{k}}\right\rangle$ are cell periodic eigenstates of the Bloch Hamiltonian

$$
H(\mathbf{k})=e^{-i \mathbf{k} \cdot r} \mathcal{H} e^{+i \mathbf{k} \cdot \mathbf{r}} .
$$

We require $\left|\psi_{n, \mathbf{k}+\mathbf{G}}\right\rangle=\left|\psi_{n, \mathbf{k}}\right\rangle$ for reciprocal-lattice vectors $\mathbf{G}$, so that the Brillouin zone in which $\mathbf{k}$ is defined is a torus. This implies $\left|u_{n, \mathbf{k}+\mathbf{G}}\right\rangle=e^{-i \mathbf{G} \cdot \mathbf{r}}\left|u_{n, \mathbf{k}}\right\rangle$. Time-reversal symmetry implies $[\mathcal{H}, \Theta]=0$, where $\Theta=\exp \left(i \pi S_{y}\right) K$ is the timereversal operator ( $S_{y}$ is spin and $K$ complex conjugation), which for spin $1 / 2$ particles satisfies $\Theta^{2}=-1$. It follows that $H(-\mathbf{k})=\Theta H(\mathbf{k}) \Theta^{-1}$.

There are special points $\mathbf{k}=\Gamma_{i}$ in the Brillouin zone which are time-reversal invariant and satisfy $-\Gamma_{i}=\Gamma_{i}+\mathbf{G}$ for a reciprocal-lattice vector $\mathbf{G}$. There are eight such points in three dimensions and four in two dimensions. At these points, $H\left(\Gamma_{i}\right)=\Theta H\left(\Gamma_{i}\right) \Theta^{-1}$, so that the eigenstates are Kramers degenerate. In the following, it will be useful to use two different notations to label the distinct $\Gamma_{i}$. (1) The eight (or four) $\Gamma_{i}$ can be indexed by three (or two) integers $n_{l}=0,1$ defined modulo 2 , which specify half a " $\bmod 2$ reciprocallattice vector,"

$$
\Gamma_{i=\left(n_{1} n_{2} n_{3}\right)}=\frac{1}{2}\left(n_{1} \mathbf{b}_{1}+n_{2} \mathbf{b}_{2}+n_{3} \mathbf{b}_{3}\right),
$$

where $\mathbf{b}_{l}$ are primitive reciprocal-lattice vectors. Two mod 2 reciprocal-lattice vectors are equivalent if they differ by twice a reciprocal-lattice vector. (2) A second notation is useful when considering a surface perpendicular to reciprocallattice vector $\mathbf{G}$. The surface time-reversal invariant momenta $\Lambda_{a}$ will be projections of pairs of $\Gamma_{i}$ which differ by $\mathbf{G} / 2$, as shown in Fig. 1. Given $\mathbf{G}$, we can define $\Gamma_{i=(a \mu)}$, such that $\Gamma_{a 1}-\Gamma_{a 2}=\mathbf{G} / 2$.

The change in the time-reversal polarization associated with a cylinder oriented along $\mathbf{G}$ due to changing the flux between two values corresponding to $\Lambda_{a}$ and $\Lambda_{b}$ can be calculated by a method analogous to the calculation of the charge polarization as a Berry's phase. ${ }^{27-30}$ In Ref. 22, we showed that the result is most simply expressed in terms of the quantities

$$
\delta_{i}=\frac{\sqrt{\operatorname{det}\left[w\left(\Gamma_{i}\right)\right]}}{\operatorname{Pf}\left[w\left(\Gamma_{i}\right)\right]}= \pm 1
$$




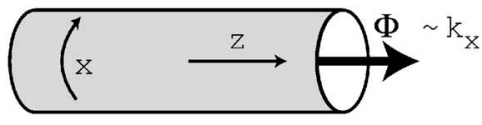

(a)

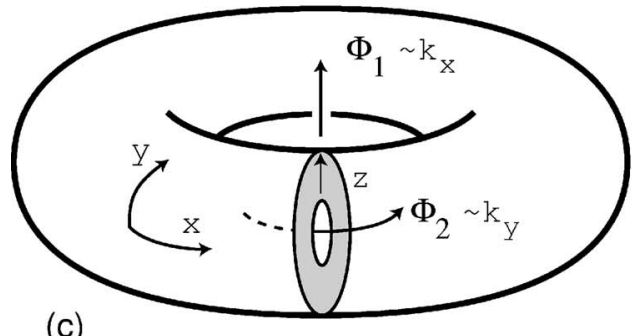

(c)

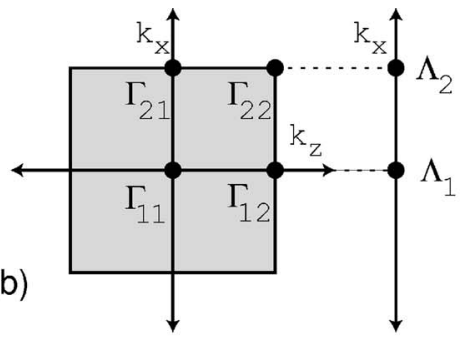

(d)

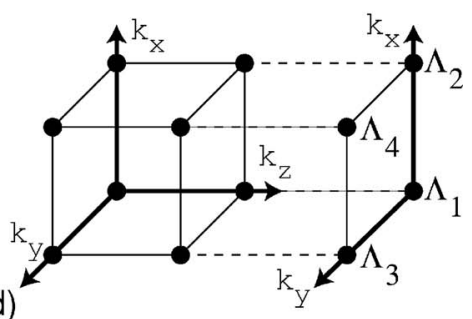

FIG. 1. (a) A two-dimensional cylinder threaded by magnetic flux $\Phi$. When the cylinder has a circumference of a single lattice constant, $\Phi$ plays the role of the edge crystal momentum $k_{x}$ in band theory. (b) The time-reversal invariant fluxes $\Phi=0$ and $h / 2 e$ correspond to edge time-reversal invariant momenta $\Lambda_{1}$ and $\Lambda_{2} . \Lambda_{a}$ are projections of pairs of the four bulk time-reversal momenta $\Gamma_{i=(a \mu)}$, which reside in the two-dimensional Brillouin zone indicated by the shaded region. (c) In three dimensions, the generalized cylinder can be visualized as a Corbino donut, with two fluxes, which correspond to the two components of the surface crystal momentum. (d) The four time-reversal invariant fluxes $\Phi_{1}, \Phi_{2}=0, h / 2 e$ correspond to the four two-dimensional surface momenta $\Lambda_{a}$. These are projections of pairs of the eight $\Gamma_{i=(a \mu)}$ that reside in the bulk $3 \mathrm{D}$ Brillouin zone.

where $w$ is the $2 N \times 2 N$ unitary matrix defined by

$$
w_{m n}(\mathbf{k}) \equiv\left\langle u_{m-\mathbf{k}}|\Theta| u_{n \mathbf{k}}\right\rangle .
$$

Since $\langle\Theta a \mid \Theta b\rangle=\langle b \mid a\rangle$ and $\Theta^{2}=-1$, the matrix $w(\mathbf{k})$ is antisymmetric for $\mathbf{k}=\Gamma_{i}$, allowing for the definition of the Pfaffian, which satisfies $\operatorname{det}[w]=\operatorname{Pf}[w]^{2}$. Given a surface $\mathbf{G}$, the time-reversal polarization associated with surface momentum $\Lambda_{a}$ was found to be

$$
\pi_{a}=\delta_{a 1} \delta_{a 2} .
$$

This formula is analogous to the expression for the charge polarization as a Berry's phase along a closed cycle in momentum space. ${ }^{28}$

Because of the square root, the sign of $\delta_{i}$ is ambiguous. However, since we require $\left|u_{n, \mathbf{k}}\right\rangle$ to be continuous, $\sqrt{\operatorname{det}[w(\mathbf{k})]}$ is defined globally throughout the Brillouin zone. The sign ambiguity then cancels for $\pi_{a}$. The existence of the global square root is guaranteed by the fact that the phase winding of $\operatorname{det}[w(\mathbf{k})]$ around any closed loop $C$ is zero, so that $\sqrt{\operatorname{det}[w(\mathbf{k})]}$ has no branch cuts. When $C$ is contractable, the vanishing phase winding follows from the continuity of $\left|u_{n, \mathbf{k}}\right\rangle$. For noncontractable loops, which can be continuously deformed to satisfy $C=-C$, it follows from the fact that $\operatorname{det}[w(-\mathbf{k})]=\operatorname{det}[w(\mathbf{k})]$.

The inherent ambiguity of the time-reversal polarization is reflected in the fact that the $\pi_{a}$ are not gauge invariant. For instance, consider a gauge transformation of the form

$$
\left|u_{n, \mathbf{k}}\right\rangle \rightarrow\left\{\begin{array}{cc}
e^{i \theta_{\mathbf{k}}}\left|u_{n, \mathbf{k}}\right\rangle & \text { for } n=1 \\
\left|u_{n, \mathbf{k}}\right\rangle & \text { for } n \neq 1 .
\end{array}\right.
$$

Under this transformation,

$$
\begin{gathered}
\operatorname{det}[w(\mathbf{k})] \rightarrow \operatorname{det}[w(\mathbf{k})] e^{-i\left(\theta_{\mathbf{k}}+\theta_{-k}\right)}, \\
\operatorname{Pf}\left[w\left(\Gamma_{i}\right)\right] \rightarrow \operatorname{Pf}\left[w\left(\Gamma_{i}\right)\right] e^{-i \theta_{\Gamma_{i}}}
\end{gathered}
$$

If we choose $\theta_{\mathbf{k}}=\mathbf{k} \cdot \mathbf{R}$ for a lattice vector $\mathbf{R}$, the Brillouinzone periodicity of $u_{n \mathbf{k}}$ is preserved. From Eq. (2.8), it is clear that $\operatorname{det}[w(\mathbf{k})]$ is unchanged. However, if $\mathbf{G} \cdot \mathbf{R}=2 \pi$, it follows that $\operatorname{Pf}\left[w\left(\Gamma_{a 1}\right)\right] \operatorname{Pf}\left[w\left(\Gamma_{a 2}\right)\right]$, and hence $\pi_{a}$ changes sign. Since this gauge transformation changes the sign of $\pi_{a}$ for all $a$, however, the product $\pi_{a} \pi_{b}$, which gives the change in the time-reversal polarization between $\Lambda_{a}$ and $\Lambda_{b}$, remains invariant. In general, the product of any four $\delta_{i}$ 's for which $\Gamma_{i}$ lie in the same plane is gauge invariant and defines a topological invariant characterizing the band structure.

In two dimensions, there are four time-reversal invariant momenta $\Gamma_{i}$ and a single $Z_{2}$ invariant, given by

$$
(-1)^{\nu}=\prod_{i=1}^{4} \delta_{i}
$$

In three dimensions there are eight time-reversal invariant points. This leads to four independent $Z_{2}$ invariants. ${ }^{13-15}$ One of these invariants, $\nu_{0}$, can be expressed as the product over all eight points,

$$
(-1)^{\nu_{0}}=\prod_{i=1}^{8} \delta_{i}
$$

The other three invariants are given by products of four $\delta_{i}$ 's for which $\Gamma_{i}$ reside in the same plane. 


$$
(-1)^{\nu_{k}}=\prod_{n_{k}=1 ; n_{j \neq k}=0,1} \delta_{i=\left(n_{1} n_{2} n_{3}\right)} .
$$

$\nu_{0}$ is clearly independent of the choice of primitive reciprocal-lattice vectors $\mathbf{b}_{k}$ in Eq. (2.3). $\left(\nu_{1} \nu_{2} \nu_{3}\right)$ are not. However, they may be viewed as components of a mod 2 reciprocal-lattice vector

$$
\mathbf{G}_{\nu}=\nu_{1} \mathbf{b}_{1}+\nu_{2} \mathbf{b}_{2}+\nu_{3} \mathbf{b}_{3} .
$$

This vector may be explicitly constructed from the $\delta_{i}$ 's as follows. A gauge transformation of the form of Eq. (2.7) can change the signs of any four $\delta_{i}$ for which the $\Gamma_{i}$ lie in the same plane. Such transformations do not change the invariants $\left(\nu_{1} \nu_{2} \nu_{3}\right)$. By a sequence of these transformations, it is always possible to find a gauge in which $\delta_{i}=-1$ for at most one nonzero $\Gamma_{i}$. Define $\Gamma^{*}=\Gamma_{i}$ if there is one such point. If there is none, then set $\Gamma^{*}=0$. In this gauge, the $\bmod 2$ reciprocal-lattice vector is $\mathbf{G}_{\nu}=2 \Gamma^{*}$. The remaining invariant $\nu_{0}$ is then determined by $\delta_{i}$ at $\Gamma_{i}=0$.

As we will explain below in Sec. II C 2, the latter invariants $\nu_{k}$ are not robust in the presence of disorder. We refer to them as "weak" topological invariants. On the other hand, $\nu_{0}$ is more fundamental and distinguishes the "strong" topological insulator.

Formulas (2.10)-(2.12) are a bit deceptive because they appear to depend solely on a local property of the wave functions. Knowledge of the global structure of $\left|u_{n \mathbf{k}}\right\rangle$, however, is necessary to construct the continuous gauge required to evaluate Eq. (2.4). The existence of globally continuous wave functions is mathematically guaranteed because the Chern number for the occupied bands vanishes due to timereversal symmetry. However, determining a continuous gauge is not always simple.

\section{Surface states}

The spectrum of surface (or edge) states as a function of momentum parallel to the surface (or edge) is equivalent to the spectrum of discrete end states of the cylinder as a function of flux. Figure 2 schematically shows two possible end state spectra as a function of momentum (or equivalently flux) along a path connecting the surface time-reversal invariant momenta $\Lambda_{a}$ and $\Lambda_{b}$. Only end states localized at one of the ends of the cylinder are shown. The shaded region gives the bulk continuum states. Time-reversal symmetry requires the end states at $\Lambda_{a}$ and $\Lambda_{b}$ to be twofold degenerate. However, there are two possible ways these degenerate states can connect up with each other. In Fig. 2(a), the Kramers pairs "switch partners" between $\Lambda_{a}$ and $\Lambda_{b}$, while in Fig. 2(b), they do not.

These two situations are distinguished by the $Z_{2}$ invariant characterizing the change in the time-reversal polarization of the cylinder when the flux is changed between the values corresponding to $\Lambda_{a}$ and $\Lambda_{b}$. Suppose that at the flux corresponding to $\Lambda_{a}$ the ground state is nondegenerate, and all levels up to and including the doublet $\varepsilon_{a 1}$ are occupied. If the flux is adiabatically changed to $\Lambda_{b}$, then for Fig. 2(a), the doublet $\varepsilon_{b 1}$ is half filled, and the ground state has a twofold Kramers degeneracy associated with the end. For Fig. 2(b),
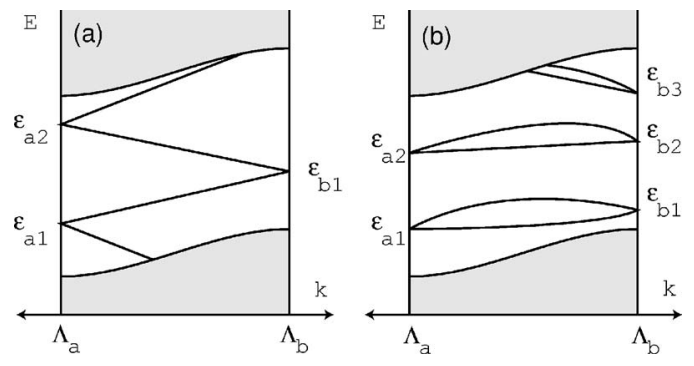

FIG. 2. Schematic representations of the surface energy levels of a crystal in either two or three dimensions as a function of surface crystal momentum on a path connecting $\Lambda_{a}$ and $\Lambda_{b}$. The shaded region shows the bulk continuum states, and the lines show discrete surface (or edge) bands localized near one of the surfaces. The Kramers degenerate surface states at $\Lambda_{a}$ and $\Lambda_{b}$ can be connected to each other in two possible ways, shown in (a) and (b), which reflect the change in time-reversal polarization $\pi_{a} \pi_{b}$ of the cylinder between those points. Case (a) occurs in topological insulators and guarantees that the surface bands cross any Fermi energy inside the bulk gap.

on the other hand, the ground state remains nondegenerate. This construction establishes the connection between the surface states and the bulk topological invariants. When $\pi_{a} \pi_{b}$ $=-1(+1)$, the surface spectrum is like Fig. 2(a) (2b).

It follows that when $\pi_{a} \pi_{b}=-1(+1)$, a generic Fermi energy inside the bulk gap will intersect an odd (even) number of surface bands between $\Lambda_{a}$ and $\Lambda_{b}$. Thus, when $\pi_{a} \pi_{b}=$ -1 , the existence of surface states is topologically protected. The details of the surface-state spectrum will depend on the Hamiltonian in the vicinity of the surface. In Fig. 2, we have assumed that surface bound states exist for all momenta. This need not be the case, since it is possible that by varying the surface Hamiltonian, the degenerate states at $\Lambda_{a}$ and $\Lambda_{b}$ can be pulled out of the gap into the bulk continuum states. This, however, does not change our conclusions regarding the number of Fermi energy crossings. When $\pi_{a} \pi_{b}=-1$, there still must exist surface band traversing the energy gap.

In the two-dimensional quantum spin-Hall phase, $\pi_{1} \pi_{2}=$ -1 , and there will be an odd number of pairs of Fermi points. ${ }^{9,10}$ In the simplest case where there is a single pair, the states at the Fermi energy will be spin filtered in the sense that the expectation value of the spin in the right and left moving states will have opposite sign. These states are robust in the presence of weak disorder and interactions because time-reversal symmetry forbids elastic backscattering. Strong interactions, however, can lead to an electronic instability that opens a gap. ${ }^{31,32}$ The resulting ground state, however, breaks time-reversal symmetry.

In three dimensions, the Kramers degenerate band crossings that occur at $\Lambda_{a}$ in the surface spectrum are twodimensional Dirac points. While such Dirac points will occur in any time-reversal invariant system with spin-orbit interactions, the nontrivial structure here arises from the way in which the Dirac points at different $\Lambda_{a}$ are connected to each other. This is determined by the relative signs of the four $\pi_{a}$ associated with any surface.

In Fig. 3, we depict four different topological classes for three-dimensional band structures labeled according to $\nu_{0}$; 


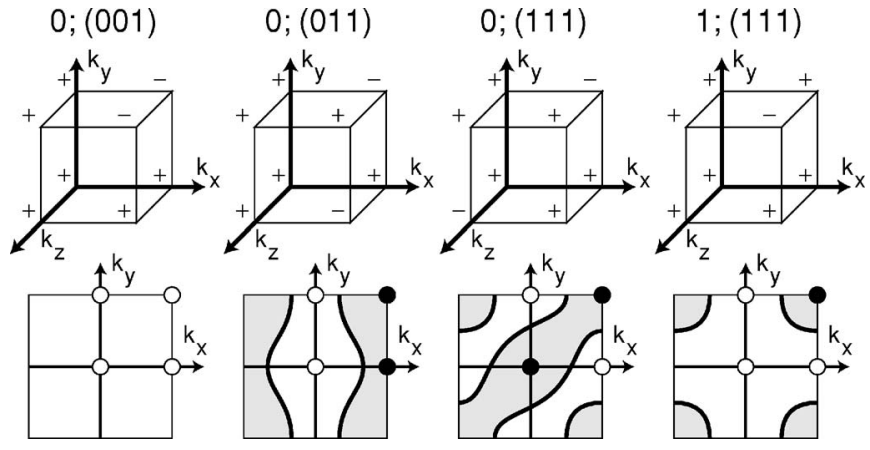

FIG. 3. Diagrams depicting four different phases indexed by $\nu_{0}$; $\left(\nu_{1} \nu_{2} \nu_{3}\right)$. The top panel depicts the signs of $\delta_{i}$ at the points $\Gamma_{i}$ on the vertices of a cube. The bottom panel characterizes the band structure of a 001 surface for each phase. The solid and open circles depict the time-reversal polarization $\pi_{a}$ at the surface momenta $\Lambda_{a}$, which are projections of pairs of $\Gamma_{i}$ that differ only in their $z$ component. The thick lines indicate possible Fermi arcs that enclose specific $\Lambda_{a}$.

$\left(\nu_{1} \nu_{2} \nu_{3}\right)$, which are characterized by different values of $\delta_{i}$ for the eight $\Gamma_{i}$, represented as the vertices of a cube in momentum space. The lower panel shows the surface Brillouin zone for a 001 surface with the four $\Lambda_{a}$ labeled by either filled or solid circles, depending on the value of $\pi_{a}=\delta_{i=(a 1)} \delta_{i=(a 2)}$. The surface band structure will resemble Fig. 2(a) on paths connecting two filled circles or two empty circles, and will resemble Fig. 2(b) on paths connecting a filled circle to an empty circle. This allows us to draw a minimal Fermi arc (denoted by the thick lines), which separates regions containing the filled circles from regions containing the empty circles.

\section{Strong topological insulator}

For the strong topological insulator, $\pi_{1} \pi_{2} \pi_{3} \pi_{4}=-1$, so that a single $\pi_{a=a^{*}}$ differs in sign from the other three. The simplest Fermi arc, shown in Fig. 3(d), thus encloses $\Lambda_{a}{ }^{*}$ once. As in the two-dimensional case, this Fermi arc cannot be eliminated. In general, time-reversal symmetry requires that any time-reversal invariant Fermi arc $C$ satisfies $C=$ $-C$. It follows that the Berry's phase acquired by an electron circling the Fermi arc can only be either 0 or $\pi$. Since the Fermi arc of the strong topological insulator encloses a single Dirac point, an electron circling the Fermi arc acquires a Berry's phase of $\pi$. Since this cannot be changed by continuous changes to the Hamiltonian, we conclude that the $\pi$ Berry's phase is a generic feature of the surface Fermi arc in the strong topological insulator phase. Such a Fermi arc defines a "topological metal," 22 which is topologically protected and, unlike an ordinary metal, cannot be depleted.

In the presence of weak disorder, the $\pi$ Berry's phase changes the sign of the weak localization correction to the conductivity and gives rise to antilocalization, as in the symplectic universality class. ${ }^{16,18}$ We suspect that in the absence of electron-electron interactions, these surface states cannot be localized even for strong disorder (provided the bulk topological phase is not destroyed). As in the 2D case, however, electron interactions can lead to a localized phase, which will necessarily break the time-reversal symmetry. $22,31,32$

In the strong topological insulator, it is possible that the Fermi energy can be tuned to intersect a single Dirac point. This is a rather unique situation, because lattice Dirac fermions are generally expected to come in pairs. ${ }^{19}$ These surface Dirac fermions are reminiscent of domain-wall fermions which have been studied in the context of lattice gauge theories. ${ }^{33}$ The surface can be viewed as an interface between the topological insulator and a conventional insulator (the vacuum). These two phases can be characterized in terms of a three-dimensional Dirac fermion, whose mass has opposite signs in the two phases (see, for example, Sec. III). The domain wall between the two is then characterized by a gapless Fermion, or zero mode, which is related to the zero energy midgap states that appear in a one-dimensional Peierls insulator at a soliton. ${ }^{34}$ However, there are some important differences between our model and the conventional applications of domain-wall fermions. (1) In our problem, there is no reason to have particle-hole symmetry, so tuning is required for the Fermi energy to be at the Dirac point. (2) The domain-wall fermion applications have often been used to model chiral fermions in even-dimensional space-time. ${ }^{33}$ Our $(2+1)$-dimensional surface Dirac fermions are not chiral. Nonetheless, they realize the $(2+1)$-dimensional "parity anomaly." ${ }^{35}$

The parity anomaly arises for a single (i.e., undoubled) species of massless Dirac fermion in $2+1$ dimensions. When the response to the electromagnetic field is naively computed in this model, one finds ${ }^{35}$

$$
J_{\lambda}= \pm \frac{e^{2}}{4 h} \epsilon_{\mu \nu \lambda} F_{\mu \nu}
$$

where $J_{\lambda}$ is the three current and $F_{\mu \nu}$ is the electromagnetic field tensor in $2+1$ dimensions. This appears "anomalous" in the sense that the electromagnetic field gives rise to currents which appear to violate the symmetries of the Dirac Hamiltonian. The sign ambiguity in Eq. (2.14) is due to the regularization procedure, in which a finite mass is included to control divergences and taken to zero at the end. The origin of the singular behavior is the subtlety of this limiting procedure.

In a magnetic field, the Dirac equation leads to a Landau level at exactly zero energy. At exactly half filling, the system is thus at a critical point separating two quantum Hall states with $\sigma_{x y}= \pm(1 / 2) e^{2} / h$. This explains the singular behavior described above. Indeed, the regulator mass term discussed above which opens a gap necessarily violates timereversal symmetry because it lifts a Kramers degeneracy. This leads to quantum Hall states even in zero applied magnetic field.

For our problem, in the absence of time-reversal symmetry-breaking perturbations, we do not expect anomalous currents to occur. However, in a magnetic field, the parity anomaly shows up in the quantum Hall effect because the surface Hall conductivity will be quantized in half integers, 


$$
\sigma_{x y}=\left(n+\frac{1}{2}\right) \frac{e^{2}}{h} .
$$

It is interesting to ask whether such a "fractional" integer quantum Hall effect could be measured. Unfortunately, in a standard transport experiment in which currents and voltages are measured by attaching leads to a sample, the $1 / 2$ cannot be directly measured. The problem is that in a slab geometry, there is no simple way to isolate the "top" surface from the "bottom" surface. The two will always be measured in parallel, and the two half integers will always add up to an integer. For such a transport experiment, there is no getting around the "fermion doubling problem." What is required is a method for measuring the current on the top surface without measuring the bottom surface. If it can be established that the currents are flowing on both surfaces, then dividing the measured Hall conductance by 2 could, in principle, demonstrate the half quantization.

A lattice realization of the parity anomaly was proposed by Fradkin and co-workers, ${ }^{36,37}$ who studied a tight-binding model for $\mathrm{PbTe}$ in the presence of a domain wall where the $\mathrm{Pb}$ and $\mathrm{Te}$ atoms are interchanged. They showed that in their model, the domain wall exhibits massless Dirac fermions and has a finite Hall conductivity even at zero magnetic field. Their model, however, is rather different from ours. In the presence of the domain wall, their Hamiltonian explicitly violates time-reversal symmetry, ${ }^{4}$ and it leads to an even number of species of Dirac fermions on the domain wall. Haldane introduced a model of the quantum Hall effect on honeycomb lattice in a periodic magnetic field. ${ }^{4,38}$ This model, which also breaks time-reversal symmetry, realizes the parity anomaly (with a single Dirac fermion) when the Hamiltonian is tuned to the transition between the quantum Hall phase and the insulator. In this model, however, the Hall conductivity is an integer.

The surface of the strong topological insulator is thus unique in that it can generate a single Dirac fermion without violating time-reversal symmetry and, in principle, exhibits the half quantized quantum Hall effect.

\section{Weak topological insulator}

When $\nu_{0}=0$, states are classified according to $\mathbf{G}_{\nu}$. We refer to the states with $\mathbf{G}_{\nu} \neq 0$ as weak topological insulators. ${ }^{15}$ $\nu_{0}=0$ implies that for any surface, the associated timereversal polarizations will satisfy $\pi_{1} \pi_{2} \pi_{3} \pi_{4}=+1$. This implies that either (1) all of the $\pi_{a}^{\prime} s$ are the same or (2) two will be positive and two will be negative. The former case occurs for surfaces $\mathbf{G}=\mathbf{G}_{\nu} \bmod 2$, where $\mathbf{G}_{\nu}$ is given in Eq. (2.13). For these surfaces, there are no topologically protected surface states. For $\mathbf{G} \neq \mathbf{G}_{\nu} \bmod 2$, two of the $\Lambda_{a}$ 's are positive and two negative. The Fermi arc encloses the two $\Lambda_{a}$ 's which have the same sign for $\pi_{a}$.

These states can be interpreted as layered twodimensional quantum spin-Hall states. To see this, consider two-dimensional planes in the quantum spin-Hall state stacked in the $z$ direction. When the coupling between the layers is zero, the electronic states will be independent of $k_{z}$. It follows that the four $\delta_{i}$ 's associated with the plane $k_{z}$ $=\pi / a$ will have product -1 and will be the same as the four associated with the plane $k_{z}=0$. The topological invariants will then be given by $\nu_{0}=0$ and $\mathbf{G}_{\nu}=(2 \pi / a) \hat{z}$. This structure will remain when weak coupling between the layers is introduced. More generally, quantum spin-Hall states stacked in the $\mathbf{G}$ direction will have $\mathbf{G}_{\nu}=\mathbf{G} \bmod 2$. This implies that quantum spin-Hall states stacked along different directions $\mathbf{G}_{1}$ and $\mathbf{G}_{2}$ are equivalent if $\mathbf{G}_{1}=\mathbf{G}_{2} \bmod 2$.

The surface states can also be understood in this manner. When the coupling between the layers is zero, it is clear that the gap in the 2D system implies that there will be no surface states on the top and bottom surfaces. On the sides, however, the Fermi points guaranteed that the edges of the twodimensional system will become straight Fermi lines in the $k_{z}$ direction. The two Fermi lines will enclose two timereversal invariant momenta, which occur at $k_{z}=0$ and $k_{z}$ $=\pi / a$, as in Fig. 3 .

Since the surface Fermi arc encloses an even number of surface time-reversal invariant momenta (and hence an even number of 2D Dirac points), it follows that the Berry's phase associated with the Fermi arc is zero. Thus, the surface states of the weak topological insulators do not enjoy the same level of topological protection as those of the strong topological insulator. Below we will argue that in the presence of disorder, the weak topological invariants lose their meaning.

\section{D. $Z_{2}$ invariant and boundary-condition sensitivity}

Niu et al. generalized the topological characterization of the integer quantum Hall effect to express the Chern invariant in terms of the sensitivity of the ground state of a bulk crystal to phase twisted boundary conditions. ${ }^{39}$ This is more fundamental than the characterization in terms of Bloch wave functions because it does not rely on the translational symmetry of the crystal. It explains the topological stability of the Hall conductance in the presence of weak disorder. In this section, we consider a corresponding generalization of the $Z_{2}$ invariant.

To do so, we consider large (but still finite) crystal with periodic boundary conditions in all but one direction. A phase twist $e^{i \theta_{i}}$ is associated with each periodic boundary condition. This has the same structure as the cylinder (and generalized cylinder) considered in Sec. II B, but now the circumferences are much larger. The fluxes now correspond to the phase twists $\theta_{i}=\Phi_{i} e / \hbar$. Since the cylinder is still finite, the discrete states associated with the ends can be characterized by their degeneracy. This allows us to characterize the change in time-reversal polarization when the phase twists are changed by $\pi$. For noninteracting electrons, the invariants characterizing a large cylinder can be deduced from the band-structure invariants by treating the entire sample to be a unit cell of an even larger crystal. It is therefore necessary to consider the effect of enlarging the unit cell on the topological invariants.

The 2D invariant $\nu$ is preserved when the unit cell is enlarged. This is easiest to see by considering the effect of doubling the unit cell on the surface spectra of Fig. 2. The effect of doubling the unit cell will be to fold the momenta $\Lambda_{a}$ and $\Lambda_{b}$ back on top of each other. If after enlarging the unit cell we add a small random potential, which lowers the 
translational symmetry, then all "accidental" degeneracies introduced by the zone folding will be lifted, while the Kramers degeneracies at the time-reversal invariant momenta will remain. It is then clear that the manner in which the degenerate states at $\Lambda_{a}$ are connected to each other is preserved when the bands are folded back. Since the invariant $\nu$ is unchanged when the unit cell is enlarged, it may be used to characterized the bulk system.

In three dimensions, the strong topological invariant $\nu_{0}$ is also invariant under enlarging the unit cell. This can be seen by noting that if the surface Fermi arc is folded back, the number of time-reversal invariant momenta enclosed is preserved modulo 2 . The weak topological invariants $\nu_{k}$, however, are not preserved by this procedure. It is possible that for a Fermi arc which encloses two time-reversal invariant momenta, the two points can be folded back on top of each other. When the two bands are coupled to each other, a gap can then open at the Fermi energy, so that the Fermi arc can disappear.

We thus conclude that the weak topological invariants are only defined for the band structure of a perfect crystal and rely on the lattice translational symmetry. For a clean system, they have important implications for the surface-state spectrum, but the topological distinction is eliminated by disorder. The strong topological invariant $\nu_{0}$, however, is robust and characterizes a bulk three-dimensional phase.

The fragility of the weak topological invariants can also be understood by considering stacked two-dimensional quantum spin-Hall states. If two identical quantum spin-Hall states are coupled together, the resulting two-dimensional system will be a simple insulator and will not have topologically protected edge states. Thus, a stack of an even number of layers will be equivalent to a stack of insulators, while a stack of an odd number of layers will define a (thicker) 2D quantum spin-Hall state. This sensitivity to the parity of the number of layers indicates that the weak topological invariants do not characterize a robust three-dimensional phase but, rather, are only properties of the band structure.

This formulation of the $Z_{2}$ invariant in terms of the change in the time-reversal polarization of a large system as a function of twisted boundary conditions can be generalized to interacting systems. This suggests that the strong topological insulator phase remains robust in the presence of weak interactions. $^{22}$

\section{III. $Z_{2}$ INVARIANTS WITH INVERSION SYMMETRY}

In this section, we show how the presence of inversion symmetry greatly simplifies the evaluation of the $Z_{2}$ invariants. We will prove Eq. (1.1), which allows topological insulators to be identified in a straightforward manner.

Suppose that the Hamiltonian $\mathcal{H}$ has an inversion center at $\mathbf{r}=0$. It follows that $[\mathcal{H}, P]=0$, or equivalently $H(-\mathbf{k})$ $=P H(\mathbf{k}) P^{-1}$, where the parity operator is defined by

$$
P\left|\mathbf{r}, s_{z}\right\rangle=\left|-\mathbf{r}, s_{z}\right\rangle .
$$

Here, $\mathbf{r}$ is the (three-dimensional) coordinate and $s_{z}$ is the spin which is unchanged by $P$ because spin is a pseudovector. The key simplification for problems with combined in- version and time-reversal symmetries is that the Berry curvature $\mathcal{F}(\mathbf{k})$ must vanish because it must simultaneously be odd under time-reversal $[\mathcal{F}(-\mathbf{k})=-\mathcal{F}(\mathbf{k})]$ and even under inversion $[\mathcal{F}(-\mathbf{k})=+\mathcal{F}(\mathbf{k})] .{ }^{17}$ The Berry curvature is given by $\nabla_{\mathbf{k}} \times \mathcal{A}(\mathbf{k})$, where the Berry's potential is

$$
\mathcal{A}(\mathbf{k})=-i \sum_{n=1}^{2 N}\left\langle u_{n, \mathbf{k}}\left|\nabla_{\mathbf{k}}\right| u_{n, \mathbf{k}}\right\rangle,
$$

where the sum is over the $2 N$ occupied bands. The fact that $\mathcal{F}(\mathbf{k})=0$ suggests that it is possible to choose a globally continuous "transverse" gauge in which $\mathcal{A}(\mathbf{k})=0$. We will show that in this special gauge, the $\delta_{i}$ defined in Eq. (2.4) are given by Eq. (1.1), so the $Z_{2}$ invariants can be easily evaluated.

The transverse gauge may be explicitly constructed as follows. In an arbitrary gauge, consider the $2 N \times 2 N$ matrix

$$
v_{m n}(\mathbf{k})=\left\langle u_{m, \mathbf{k}}|P \Theta| u_{n, \mathbf{k}}\right\rangle .
$$

Since $\langle a \mid b\rangle=\langle\Theta b \mid \Theta a\rangle$ and $\Theta^{2}=-1$, it follows that $v(\mathbf{k})$ is antisymmetric. Since $[P \Theta, H(\mathbf{k})]=0, v(\mathbf{k})$ is unitary. Thus, the Pfaffian of $v(\mathbf{k})$ is defined and has unit magnitude. The phase of $\operatorname{Pf}[v(\mathbf{k})]$ depends on the gauge, and its gradient is related to $\mathcal{A}(\mathbf{k})$ :

$$
\mathcal{A}(\mathbf{k})=-\frac{i}{2} \operatorname{Tr}\left[v(\mathbf{k})^{\dagger} \nabla_{\mathbf{k}} v(\mathbf{k})\right]=-i \nabla_{\mathbf{k}} \log \operatorname{Pf}[v(\mathbf{k})] .
$$

The first equality is derived by differentiating Eq. (3.3) and using the unitarity of $v(\mathbf{k})$. The second equality follows from the facts that $\operatorname{det}[v]=\operatorname{Pf}[v]^{2}$ and $\nabla_{k} \log \operatorname{det}[v]$ $=\operatorname{Tr}\left[\nabla_{k} \log v(\mathbf{k})\right]=\operatorname{Tr}\left[v^{\dagger}(\mathbf{k}) \nabla_{\mathbf{k}} v(\mathbf{k})\right]$.

To set $\mathcal{A}(\mathbf{k})=0$, we thus adjust the phase of $\left|u_{n \mathbf{k}}\right\rangle$ to make

$$
\operatorname{Pf}[v(\mathbf{k})]=1 .
$$

This can be done, for instance, by a transformation of the form of Eq. (2.7), under which $\operatorname{Pf}[v(\mathbf{k})] \rightarrow \operatorname{Pf}[v(\mathbf{k})] e^{-i \theta_{\mathbf{k}}}$. In this gauge, the problem of continuing $\sqrt{\operatorname{det}[w(\mathbf{k})]}$ between the $\Gamma_{i}$ in Eq. (2.4) is eliminated because $\operatorname{det}[w(\mathbf{k})]=1$ for all $\mathbf{k}$. This can be seen by noting that $v(\mathbf{k})$ has the property $v(-\mathbf{k})=w(\mathbf{k}) v(\mathbf{k})^{*} w(\mathbf{k})^{T}$ and using the identity $\operatorname{Pf}\left[X A X^{T}\right]$ $=\operatorname{Pf}[A] \operatorname{det}[X]$.

All that remains for Eq. (2.4) is to evaluate $\operatorname{Pf}\left[w\left(\Gamma_{i}\right)\right]$. To this end, we note that

$$
w_{m n}\left(\Gamma_{i}\right)=\left\langle\psi_{m, \Gamma_{i}}|P(P \Theta)| \psi_{n \Gamma_{i}}\right\rangle .
$$

Here, we have used $P^{2}=1$, along with the antilinearity of $\Theta$, which allows us to replace $\left|u_{n \Gamma_{i}}\right\rangle$ by $\left|\psi_{n \Gamma_{i}}\right\rangle=\left|\psi_{n-\Gamma_{i}}\right\rangle$ in Eq. (2.5). Since $[\mathcal{H}, P]=0,\left|\psi_{n \Gamma_{i}}\right\rangle$ is an eigenstate of $P$ with eigenvalue $\xi_{n}\left(\Gamma_{i}\right)= \pm 1$. After changing $\left|\psi_{n \Gamma_{i}}\right\rangle$ back to $\left|u_{n \Gamma_{i}}\right\rangle$, it follows that

$$
w_{m n}\left(\Gamma_{i}\right)=\xi_{m}\left(\Gamma_{i}\right) v_{m n}\left(\Gamma_{i}\right) .
$$

The Pfaffian can be deduced from the following argument, which uses the fact that the $\operatorname{Pf}[w]$ will be a polynomial in $\xi_{n}$. First, note that 


$$
\operatorname{Pf}[w]^{2}=\operatorname{det}[w]=\operatorname{det}[v] \prod_{n=1}^{2 N} \xi_{n} .
$$

Due the Kramers degeneracy, the distinct states $\left|u_{2 m, \Gamma_{i}}\right\rangle$ and $\left|u_{2 m+1, \Gamma_{i}}\right\rangle \equiv \Theta\left|u_{2 m, \Gamma_{i}}\right\rangle$ share the same parity eigenvalue. Thus, each eigenvalue appears twice in the product in Eq. (3.8). Taking the square root, we find

$$
\operatorname{Pf}[w]=\operatorname{Pf}[v] \prod_{m=1}^{N} \xi_{2 m} .
$$

The sign of the square root is fixed by the special case in which all $\xi_{n}=1$, so that $w=v$. Since $\operatorname{Pf}[v]=1$, we conclude that in the transverse gauge,

$$
\delta_{i}=\prod_{m=1}^{N} \xi_{2 m}\left(\Gamma_{i}\right)
$$

Equation (3.10) is a central result of this paper. It means that with inversion symmetry, the $Z_{2}$ topological invariants can be deduced from the knowledge of the parity of each pair of Kramers degenerate occupied energy bands at the four (or eight in three dimensions) time-reversal and parity invariant points in the Brillouin zone. This provides a simple method for determining the topological phase of any inversion symmetric insulator, without having to know about the global properties of the energy bands.

In Eq. (3.10), it appears as though each of the four (or eight) $\delta_{i}$ have gauge independent meaning and thus provide extra topological invariants in addition to the one (or four) time-reversal symmetry based invariant discussed in Sec. II B. These extra invariants, however, rely on the presence of inversion symmetry and lose their meaning in the presence of surfaces, disorder, or other perturbations which violate inversion symmetry. In contrast, the invariants obtained from the product of four $\delta_{i}$ 's do not rely on inversion symmetry for their existence. They depend only on time-reversal symmetry, so they retain their value in the presence of inversion symmetry-breaking perturbations.

\section{TIGHT-BINDING MODELS}

In this section, we construct a class of inversion symmetric tight-binding models that exhibit topological insulating states and apply the method presented in Sec. III to determine their topological classes. We will consider minimal models with four bands which result from four degrees of freedom per unit cell. We will focus on lattices in which the unit cell can be chosen to be inversion symmetric. We will see that this latter assumption makes the analysis of the topological phases particularly simple. While this assumption can always be satisfied for continuum models, it rules out certain inversion symmetric lattice models, such as the rocksalt lattice. It is satisfied, however, for the specific examples we will consider.

In Sec. IV A, we study the general structure of this class of models, and then in Secs. IV B and IV C consider the specific examples of the honeycomb lattice of graphene and the diamond lattice. In Sec. IV D, we analyze a model for
$\mathrm{HgTe} / \mathrm{CdTe}$ quantum wells introduced recently by Bernevig et al. $^{40}$

\section{A. General model}

We assume that each unit cell associated with Bravais lattice vector $\mathbf{R}$ has four states $|\mathbf{R}, n\rangle$. If the unit cell is parity invariant, then the parity operator $P$ may be represented as a $4 \times 4$ matrix as

$$
P|\mathbf{R}, n\rangle=\sum_{m} \hat{P}_{n m}|-\mathbf{R}, m\rangle .
$$

In Secs. IV B and IV C, we will consider examples in which each unit cell consists of two sublattices (denoted by Pauli matrix $\sigma^{z}$ ) which are interchanged by inversion and two spin degrees of freedom (denoted by $s^{z}$ ). Therefore,

$$
\hat{P}=\sigma^{x} \otimes I,
$$

where $I$ is the identity for the spin indices. In Sec. IV D, $\hat{P}$ will have a slightly different form. The time-reversal operator acting on the four component basis states is represented by

$$
\hat{\Theta}=i\left(I \otimes s^{y}\right) K,
$$

where $K$ is complex conjugation and $I$ acts on the orbital indices.

Given a lattice Hamiltonian $\mathcal{H}$ in the $|\mathbf{R}, n\rangle$ basis, we now consider the Bloch Hamiltonian

$$
H(\mathbf{k})=e^{i \mathbf{k} \cdot \mathbf{R}} \mathcal{H} e^{-i \mathbf{k} \cdot \mathbf{R}},
$$

which for lattice periodic Bloch functions now becomes a $4 \times 4$ matrix. Note that this transformation is slightly different than the standard transformation of a tight-binding model for which $\mathbf{R}$ in Eq. (4.4) is replaced by $\mathbf{r}=\mathbf{R}+\mathbf{d}_{n}$, where $\mathbf{d}_{n}$ is a basis vector. The difference is a choice of gauge. With this choice, $H(\mathbf{k})$ has the properties $H(\mathbf{k}+\mathbf{G})=H(\mathbf{k})$ and $H(-\mathbf{k})$ $=\hat{P} H(\mathbf{k}) \hat{P}^{-1}$. Thus, at the time-reversal invariant momenta, $\left[H\left(\mathbf{k}=\Gamma_{i}\right), \hat{P}\right]=0$.

It is convenient to express the $4 \times 4$ matrix $H(\mathbf{k})$ in terms of the identity $I$, five Dirac matrices $\Gamma^{a}$, and their ten commutators $\Gamma^{a b}=\left[\Gamma^{a}, \Gamma^{b}\right] /(2 i){ }^{41}$ The Dirac matrices satisfy the Clifford algebra, $\Gamma^{a} \Gamma^{b}+\Gamma^{b} \Gamma^{a}=2 \delta_{a b} I$. In this section, in order to avoid confusion of notation, the Dirac matrices $\Gamma^{a}$ will always appear with a superscript, and the time-reversal invariant momenta will always be written as $\mathbf{k}=\Gamma_{i}$.

The choice of Dirac matrices is not unique. For example, in Ref. 10, the Dirac matrices were chosen to be even under time reversal, $\hat{\Theta} \Gamma^{a} \hat{\Theta}^{-1}=\Gamma^{a}$. In the presence of both inversion and time-reversal symmetries, it is more convenient to choose the Dirac matrices to be even under $\hat{P} \hat{\Theta}$. Given the form of $\hat{P}$ and $\hat{\Theta}$, the five matrices are

$$
\Gamma^{(1,2,3,4,5)}=\left(\sigma^{x} \otimes I, \sigma^{y} \otimes I, \sigma^{z} \otimes s^{x}, \sigma^{z} \otimes s^{y}, \sigma^{z} \otimes s^{z}\right) .
$$

With this choice of Dirac matrices, the commutators are odd under $\hat{P} \hat{\Theta},(\hat{P} \hat{\Theta}) \Gamma^{a b}(\hat{P} \hat{\Theta})^{-1}=-\Gamma^{a b}$. Note that $\Gamma^{1}=\hat{P}$. It follows that 


$$
\hat{\Theta} \Gamma^{a} \hat{\Theta}^{-1}=\hat{P} \Gamma^{a} \hat{P}^{-1}=\left\{\begin{array}{lc}
+\Gamma^{a} & \text { for } a=1 \\
-\Gamma^{a} & \text { for } a \neq 1 .
\end{array}\right.
$$

Time-reversal and inversion symmetries imply that $[H(\mathbf{k}), \hat{P} \hat{\Theta}]=0$. The most general Hamiltonian matrix is then

$$
H(\mathbf{k})=d_{0}(\mathbf{k}) I+\sum_{a=1}^{5} d_{a}(\mathbf{k}) \Gamma^{a} .
$$

Written in this form, the energy eigenvalues naturally come in Kramers degenerate pairs with energy

$$
E(\mathbf{k})=d_{0}(\mathbf{k}) \pm \sqrt{\sum_{a} d_{a}(\mathbf{k})^{2}} .
$$

At the time-reversal invariant points $\mathbf{k}=\Gamma_{i}$, only $\Gamma^{1}=\hat{P}$ is even under $\hat{P}$ and $\hat{\Theta}$. Therefore,

$$
H\left(\mathbf{k}=\Gamma_{i}\right)=d_{0}\left(\mathbf{k}=\Gamma_{i}\right) I+d_{1}\left(\mathbf{k}=\Gamma_{i}\right) \hat{P} .
$$

The parity eigenvalues $\xi_{n}$ for the states at $\mathbf{k}=\Gamma_{i}$ are given by the eigenvalues of $\hat{P}$. It then follows from Eq. (1.1) that, provided there is an energy gap throughout the Brillouin zone, the $Z_{2}$ invariants characterizing the valence band are determined by

$$
\delta_{i}=-\operatorname{sgn}\left(d_{1}\left(\mathbf{k}=\Gamma_{i}\right)\right) .
$$

We will use the above equation to determine the topological class of specific tight-binding models in the following.

\section{B. Graphene}

Graphene consists of a honeycomb lattice of carbon atoms with two sublattices. A tight-binding model which incorporates the symmetry allowed spin-orbit interactions was introduced in Refs. 9 and 10,

$$
H=t \sum_{\langle i j\rangle} c_{i}^{\dagger} c_{j}+i \lambda_{S O} \sum_{\langle i j\rangle\rangle} c_{i}^{\dagger} \mathbf{s} \cdot \hat{\mathbf{e}}_{i j} c_{j} .
$$

The first term is a nearest-neighbor hopping term, and the second term is spin-orbit interaction which involves spin dependent second-neighbor hopping. This term is written in a way which can easily be generalized to three dimensions. $\mathbf{s}$ is the spin, and we have defined the unit vector

$$
\hat{\mathbf{e}}_{i j}=\frac{\mathbf{d}_{i j}^{1} \times \mathbf{d}_{i j}^{2}}{\left|\mathbf{d}_{i j}^{1} \times \mathbf{d}_{i j}^{2}\right|},
$$

where $\mathbf{d}_{i j}^{1}$ and $\mathbf{d}_{i j}^{2}$ are bond vectors along the two bonds the electron traverses when going from site $j$ to $i$. Thus, $\hat{\mathbf{e}}_{i j} \cdot \mathbf{S}$ $= \pm s^{z}$.

Choosing the unit cell shown in Fig. 4, the Hamiltonian matrix $H(\mathbf{k})$ can be determined using Eq. (4.4) and expressed in terms of Dirac matrices as in Eq. (4.7). The coefficients $d_{a}(\mathbf{k})$ are displayed in Table I. The time-reversal invariant momenta, in the notation of Eq. (2.3), occur at $\mathbf{k}=\Gamma_{i=\left(n_{1} n_{2}\right)}$ for $n_{l}=0,1$. The Hamiltonian at these points can be deduced by noting that at $\mathbf{k}=\Gamma_{i=\left(n_{1} n_{2}\right)}, x_{l} \equiv \mathbf{k} \cdot \mathbf{a}_{l}=n_{l} \pi . \Gamma_{i=(00)}$ is commonly referred to as the $\Gamma$ point. The other three, which are
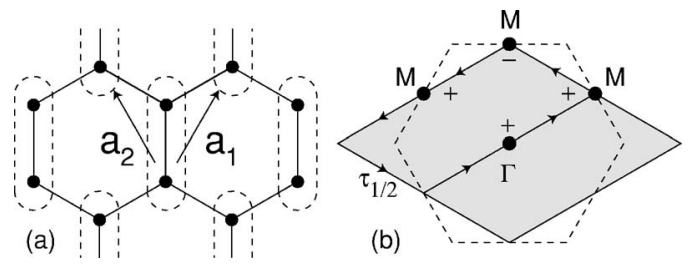

FIG. 4. (a) Honeycomb lattice of graphene, with a unit cell indicated by the dashed lines. (b) Brillouin zone with the values of $\delta_{i}$ associated with the time-reversal invariant momenta labeled. $\tau_{1 / 2}$ describes the loop enclosing half the zone used in Eq. (4.13).

equivalent under threefold rotations, are called the $M$ points. Using Eqs. (1.1), (1.2), and (4.10), it is then straightforward to see that $\delta_{i=(00)}=\delta_{i=(10)}=\delta_{i=(01)}=-1$ while $\delta_{i=(11)}=+1$. The product is negative, so $\nu=1$, and provided that the energy gap is finite throughout the Brillouin zone, the system is a topological insulator in the quantum spin-Hall phase. The finite gap follows from the fact that $d_{0}(\mathbf{k})=0$ and there are no values of $\mathbf{k}$ for which all $d_{a}(\mathbf{k})=0$.

The reason the three $M$ points are not all the same is that the center of inversion defined by our unit cell is at the center of a bond, which does not have the threefold rotational symmetry. By choosing a different unit cell, with inversion center at the center of a hexagon, the $M$ points would be equivalent. Our conclusion about the topological class, however, remains the same.

It is interesting to note that the value of $\delta_{i}$ does not appear to have anything to do with the spin-orbit interaction. The role that the spin-orbit interaction plays is simply to ensure that the energy gap is finite everywhere in the Brillouin zone. We will now argue for a parity and time-reversal invariant system that if the spin-orbit interaction is absent, then the negative product of $\delta_{i}$ implies that the energy gap must vanish somewhere in the Brillouin zone. This gives insight into the topological stability of the Dirac points in graphene in the absence of spin-orbit interactions.

We prove this by contradiction. In the absence of the spinorbit interaction, we can consider spinless fermions. Suppose there is a finite gap everywhere, and the valence band is well defined throughout the Brillouin zone. Then, on the one hand, the Berry curvature $\mathcal{F}=\nabla \times \mathcal{A}$ is identically zero due to inversion and time-reversal symmetries. On the other hand, we will show that the Berry's phase for the path $\tau_{1 / 2}$ shown in Fig. 4 which encloses half the Brillioun zone satisfies

TABLE I. Parameters for tight-binding model of graphene with $x_{l}=\mathbf{k} \cdot \mathbf{a}_{l}$ in a notation slightly different from Ref. 10 .

\begin{tabular}{lc}
\hline$d_{0}$ & 0 \\
$d_{1}$ & $t\left[1+\cos x_{1}+\cos x_{2}\right]$ \\
$d_{2}$ & $t\left[\sin x_{1}+\sin x_{2}\right]$ \\
$d_{3}$ & 0 \\
$d_{4}$ & 0 \\
$d_{5}$ & $2 \lambda_{S O}\left[\sin x_{1}-\sin x_{2}-\sin \left(x_{1}-x_{2}\right)\right]$ \\
\hline \hline
\end{tabular}


TABLE II. Parameters for diamond lattice tight-binding model, with $x_{k}=\mathbf{k} \cdot \mathbf{a}_{k}$.

\begin{tabular}{lc}
\hline \hline$d_{0}$ & 0 \\
$d_{1}$ & $t+\delta t_{1}+t\left[\cos x_{1}+\cos x_{2}+\cos x_{3}\right]$ \\
$d_{2}$ & $t\left[\sin x_{1}+\sin x_{2}+\sin x_{3}\right]$ \\
$d_{3}$ & $\lambda_{S O}\left[\sin x_{2}-\sin x_{3}-\sin \left(x_{2}-x_{1}\right)+\sin \left(x_{3}-x_{1}\right)\right]$ \\
$d_{4}$ & $\lambda_{S O}\left[\sin x_{3}-\sin x_{1}-\sin \left(x_{3}-x_{2}\right)+\sin \left(x_{1}-x_{2}\right)\right]$ \\
$d_{5}$ & $\lambda_{S O}\left[\sin x_{1}-\sin x_{2}-\sin \left(x_{1}-x_{3}\right)+\sin \left(x_{2}-x_{3}\right)\right]$ \\
\hline \hline
\end{tabular}

$$
\exp \left[i \oint_{\tau_{1 / 2}} \mathcal{A}(\mathbf{k}) \cdot d \mathbf{k}\right]=\delta_{1} \delta_{2} \delta_{3} \delta_{4} .
$$

Thus, if $\delta_{1} \delta_{2} \delta_{3} \delta_{4}=-1$, it would violate Stoke's theorem and leads to a contradiction. The $\pi$ Berry's phase thus requires that there either be a Dirac point in each half of the Brillouin zone or a Fermi arc enclosing a Dirac point.

To obtain Eq. (4.13) for spinless electrons, we consider the unitary matrix

$$
m_{i j}(\mathbf{k})=\left\langle u_{i,-\mathbf{k}}|P| u_{j, \mathbf{k}}\right\rangle,
$$

which is related to the Berry's potential via $\nabla_{k} \log \operatorname{det}[m(\mathbf{k})]=-i[\mathcal{A}(\mathbf{k})+\mathcal{A}(-\mathbf{k})]$. Equation (4.13) is then obtained by breaking the line integral into segments connecting the time-reversal invariant momenta and using the fact that $\operatorname{det}\left[m\left(\mathbf{k}=\Gamma_{i}\right)\right]=\delta_{i}$.

\section{Diamond lattice}

We now consider the tight-binding model on a diamond lattice introduced in Ref. 15. This model exhibits both weak and strong topological insulator phases.

The diamond structure consists of two interpenetrating face-centered-cubic lattices displaced from each other by a basis vector $\mathbf{d}=a(1,1,1) / 4$. The primitive translation vectors $\mathbf{a}_{1}, \mathbf{a}_{2}$, and $\mathbf{a}_{3}$ are $a(0,1,1) / 2, a(1,0,1) / 2$, and $a(1,1,0) / 2$, respectively. Our model has the same form as Eq. (4.11) and includes a nearest-neighbor hopping term as well as a second-neighbor spin-orbit interaction.

It turns out that with this spin-orbit interaction term, the valence bands and conduction bands meet at 3D Dirac points at the three inequivalent $X$ points on the 100, 010, and 001 faces of the Brillouin zone. In order to lift the degeneracy and obtain a gapped phase, we introduced a distortion, which changes the nearest-neighbor hopping amplitudes. For simplicity, we will focus here on a distortion in the 111 direction, which changes the nearest-neighbor bond in the 111 direction but leaves the other three bonds alone. The resulting model can be expressed in the form of Eq. (4.7), and the resulting $d_{a}(\mathbf{k})$ are listed in Table II. For $\lambda_{S O}, \delta t \neq 0$, the gap is finite throughout the Brillouin zone.

As in the previous section, the time-reversal invariant momenta occur at $\mathbf{k}=\Gamma_{i=\left(n_{1} n_{2} n_{3}\right)}$ as in Eq. (2.3). At these points, $x_{l} \equiv \mathbf{k} \cdot \mathbf{a}_{l}=n_{l} \pi$. At the $\Gamma$ point, $\mathbf{k}=0,\left(n_{1} n_{2} n_{3}\right)=(000)$. The three inequivalent $X$ points [at $\mathbf{k}=(2 \pi / a)(1,0,0)$ and related points] have $\left(n_{1} n_{2} n_{3}\right)=(011),(101)$, and (110). The four inequivalent $L$ points [at $\mathbf{k}=(\pi / a)(1,1,1)$ and related points] have $\left(n_{1} n_{2} n_{3}\right)=(100),(010),(001)$, and (111). The 111 distortion makes the first three $L$ points distinct from the fourth, which will be referred to as $T$.

From Table II, we can deduce the sign of $d_{1}(\mathbf{k})$ and, hence, $\delta_{i}$ at these points. We find $\delta_{i}=-1$ at $\Gamma$ and the three $L$ points, while $\delta_{i}=+1$ at $T$. At the $X$ points, $\delta_{i}=-\operatorname{sgn}\left(\delta t_{1}\right)$. Combining these, we then find that

$$
\left(\nu_{0} ; \nu_{1} \nu_{2} \nu_{3}\right)= \begin{cases}(1 ; 111) & \text { for } \delta t_{1}>0 \\ (0 ; 111) & \text { for } \delta t_{1}<0 .\end{cases}
$$

When the 111 distorted bond is stronger than the other three bonds, so that the system is dimerized, the system is a strong topological insulator. When the 111 bond is weaker than the other three, so that the system is layered, it is a weak topological insulator with $\mathbf{G}_{\nu}=(2 \pi / a)(1,1,1)$, which can be viewed as two-dimensional quantum spin-Hall states stacked in the 111 direction.

In Ref. 15, we computed the two-dimensional band structure for the diamond lattice model in a slab geometry. The results displayed the expected surface states, which behave according to the general principles discussed in Sec. II C.

\section{Bernevig-Hughes-Zhang model}

After this paper was originally submitted, an interesting proposal appeared for the 2D quantum spin-Hall effect in quantum well structures in which a layer of $\mathrm{HgTe}$ is sandwiched between crystals of CdTe. ${ }^{40}$ Bernevig-Hughes-Zhang (BHZ) showed that for an appropriate range of well thickness, the HgTe layer exhibits an inverted band structure, where the $s$ and $p$ levels at the conduction- and valence-band edges are interchanged. In this inverted regime, the structure exhibits a 2D quantum spin-Hall effect. BHZ introduced a simple four band tight-binding model which captures this effect. Though real HgTe does not have inversion symmetry, their toy model does. In this section, we analyze this model and directly evaluate the $Z_{2}$ topological invariant using Eq. (1.1).

BHZ considered a four band model on a square lattice in which each site has two $s_{1 / 2}$ states $|s, \uparrow\rangle$ and $|s, \downarrow\rangle$ and two of the crystal field split $p_{3 / 2}$ states (with $m_{j}= \pm 3 / 2$ ), $\mid p_{x}$ $\left.+i p_{y}, \uparrow\right\rangle$ and $\left|p_{x}-i p_{y}, \downarrow\right\rangle$. The Hamiltonian is

$$
H=\sum_{i, \sigma, \alpha} \varepsilon_{\alpha} c_{i \alpha \sigma}^{\dagger} c_{i \alpha \sigma}-\sum_{i a \sigma \alpha \beta} t_{a \sigma, \alpha \beta} c_{i+a \alpha \sigma}^{\dagger} c_{i \beta \sigma},
$$

where $a$ labels the four nearest neighbors, $\sigma= \pm 1$ describes the spin, and $\alpha, \beta=s, p$ is the orbital index. The hopping term involves the matrix

$$
t_{a \sigma}=\left(\begin{array}{cc}
t_{s s} & t_{s p} e^{i \sigma \theta_{a}} \\
t_{s p} e^{-i \sigma \theta_{a}} & -t_{p p}
\end{array}\right),
$$

where $\theta_{a}$ gives the angle of nearest-neighbor bond $a$ with the $x$ axis.

As in Sec. IV A, it is convenient to express this Hamiltonian in the form of Eq. (4.7) using Dirac matrices which are even under $\hat{P} \hat{\Theta}$. The form of the parity operator, however, is slightly different in this model, and Eq. (4.2) is replaced by 
TABLE III. Parameters for the BHZ model with $x_{k}=\mathbf{k} \cdot \mathbf{a}_{k}$.

\begin{tabular}{lc}
\hline \hline$d_{0}$ & $\left(\varepsilon_{s}+\varepsilon_{p}\right) / 2-\left(t_{s s}-t_{p p}\right)\left(\cos x_{1}+\cos x_{2}\right)$ \\
$d_{1}$ & $\left(\varepsilon_{s}-\varepsilon_{p}\right) / 2-\left(t_{s s}+t_{p p}\right)\left(\cos x_{1}+\cos x_{2}\right)$ \\
$d_{2}$ & $2 t_{s p} \sin x_{1}$ \\
$d_{3}$ & 0 \\
$d_{4}$ & 0 \\
$d_{5}$ & $2 t_{s p} \sin x_{2}$ \\
\hline \hline
\end{tabular}

$$
\hat{P}=\sigma^{z} \otimes I
$$

where $\sigma^{z}=+1(-1)$ describes $s(p)$ states. The Dirac matrices are then the same as Eq. (4.5), except that $\sigma^{x}$ and $\sigma^{z}$ are interchanged. The coefficients of these new Dirac matrices for this model are displayed in Table III.

The analysis between Eqs. (4.6) and (4.10) proceeds exactly the same as before, and $\delta_{i}=-\operatorname{sgn}\left[d_{1}\left(\mathbf{k}=\Gamma_{i}\right)\right]$. We conclude that for $\mathbf{k}=(\pi / a)\left(n_{1}, n_{2}\right)$,

$$
\delta_{i=\left(n_{1} n_{2}\right)}=-\operatorname{sgn}\left\{\frac{\varepsilon_{s}-\varepsilon_{p}}{2}-\left(t_{s s}+t_{p p}\right)\left[(-1)^{n_{1}}+(-1)^{n_{2}}\right]\right\} .
$$

For $\varepsilon_{s}-\varepsilon_{p}>4\left(t_{s s}+t_{p p}\right)$, all of the $\delta_{i=\left(n_{1} n_{2}\right)}$ are negative, so that the product $\nu=+1$. The system is a simple insulator. In this regime, the bands have a conventional ordering throughout the Brillouin zone, with the $s$ states in the conduction band and the $p$ states in the valence band. For $\varepsilon_{s}-\varepsilon_{p} \lesssim 4\left(t_{s s}+t_{p p}\right)$, the bands near $\mathbf{k}=0$ become inverted, and $\delta_{i=(00)}$ becomes positive, signaling a transition into the quantum spin-Hall phase in which $\nu=-1$.

\section{TOPOLOGICAL PHASES IN SPECIFIC MATERIALS}

In this section, we apply our method for evaluating the topological invariants to identify specific three-dimensional materials that should exhibit a strong topological insulating phase.

\section{A. Bismuth-antimony alloy}

$\mathrm{Bi}$ and $\mathrm{Sb}$ are group- $\mathrm{V}$ semimetals in which there is a finite direct energy gap throughout the Brillouin zone but a negative indirect gap due to band overlap. They have very close lattice parameters and form the solid alloy $\mathrm{Bi}_{1-x} \mathrm{Sb}_{x}{ }^{42,43}$ For $0.07<x<0.22$, the indirect gap becomes positive, leading to semiconducting behavior, with a maximum energy gap of order $30 \mathrm{meV}$ for $x=0.18$. In this section, we will argue, based on the known band structure of these materials, that this alloy is a strong topological insulator, which will have topological metal surface states.

Bulk bismuth and antimony have the rhombohedral $A 7$ structure, which consists of two interpenetrating, facecentered-cubic lattices which are displaced in the 111 direction and slightly distorted in the 111 direction. In bismuth, ${ }^{44,45}$ the valence band crosses the Fermi energy in the vicinity of the $T$ point, which is located on the 111 face of the Brillouin zone, giving rise to a small pocket of holes.
TABLE IV. Symmetry labels for the five valence bands of bismuth and antimony at eight time reversal invariant momenta according to Ref. 45. The parity eigenvalues can be read from $+/-$ and $s / a$. Using Eqs (1.1) and (1.2), they determine the topological class. The indices (111) define a mod 2 vector [Eq. (2.13) in the direction of the $T$ point.

\begin{tabular}{lcc}
\hline \hline \multicolumn{3}{c}{ Bismuth } \\
$1 \Gamma$ & $\Gamma_{6}^{+} \Gamma_{6}^{-} \Gamma_{6}^{+} \Gamma_{6}^{+} \Gamma_{45}^{+}$ & - \\
$3 L$ & $L_{s} L_{a} L_{s} L_{a} L_{a}$ & - \\
$3 X$ & $X_{a} X_{s} X_{s} X_{a} X_{a}$ & - \\
$1 T$ & $T_{6} T_{6}^{+} T_{6} T_{6}^{+} T_{45}^{-}$ & - \\
& $Z_{2}$ class & $(0 ; 000)$ \\
& Antimony & - \\
& $\Gamma_{6}^{+} \Gamma_{6}^{-} \Gamma_{6}^{+} \Gamma_{6}^{+} \Gamma_{45}^{+}$ & + \\
$3 L$ & $L_{s} L_{a} L_{s} L_{a} L_{s}$ & - \\
$3 X$ & $X_{a} X_{s} X_{s} X_{a} X_{a}$ & - \\
$1 T$ & $T_{6} T_{6}^{+} T_{6} T_{6}^{+} T_{45}^{-}$ & $(1 ; 111)$ \\
& $Z_{2}$ class \\
\hline \hline
\end{tabular}

The conduction band crosses the Fermi energy near the three equivalent $L$ points, which reside at the other three-body center zone faces, giving rise to pockets of electrons. At the $L$ points, the bottom of the conduction band, which has $L_{s}$ symmetry, is only slightly higher in energy than the next lower band, which has $L_{a}$ symmetry. In antimony, ${ }^{45}$ the electrons are again near the $L$ point. However, unlike bismuth, the bottom of the conduction band has $L_{a}$ symmetry. The holes are not at the $T$ point but, rather, at the lower-symmetry $H$ point.

Despite the fact that bismuth and antimony have negative indirect gaps, the finite direct gap throughout the Brillouin zone allows for the topological characterization of the valence energy bands. Since both bismuth and antimony have inversion symmetry, we can apply Eqs. (1.1) and (1.2) by reading off the parity eigenvalues $\xi_{n}\left(\Gamma_{i}\right)$ from published band structures. $^{44,45}$ In Table IV, we display the symmetry labels for the five occupied valence bands at the eight time-reversal invariant momenta $\left(\Gamma_{i}=\Gamma, T\right.$, and the three equivalent $L$ and $X$ points). The parity eigenvalue $\xi_{n}\left(\Gamma_{i}\right)$ can be read from the superscripts \pm or the subscripts $s / a=+/-$. (For an explanation of this notation, see Ref. 46). The right column displays the product $\delta_{i}$ from Eq. (1.1). Based on these data, we conclude that the valence band of bismuth is equivalent to that of a conventional insulator, while the valence band of antimony is that of a strong topological insulator. The difference between the two is due to the inversion between the $L_{s}$ and $L_{a}$ bands.

The evolution of the band structure of the alloy $\mathrm{Bi}_{1-x} \mathrm{Sb}_{x}$ as a function of $x$ has been well studied ${ }^{42,43,47}$ and is summarized in Fig. 5. As the $\mathrm{Sb}$ concentration is increased, two things happen. First, the gap between the $L_{s}$ and $L_{a}$ bands decreases. At $x=0.04$, the bands cross and the gap reopens with the inverted ordering. Second, the top of the valence band at $T$ comes down in energy and crosses the bottom of the conduction band at $x=0.07$. At this point, the indirect gap becomes positive, and the alloy is a semiconductor. At $x$ 


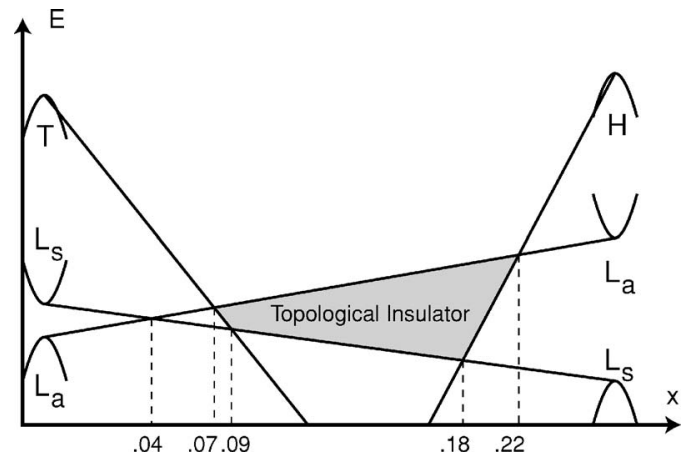

FIG. 5. Schematic representation of band energy evolution of $\mathrm{Bi}_{1-x} \mathrm{Sb}_{x}$ as a function of $x$. Adapted from Ref. 43.

$=0.09$ the $T$ valence band clears the $L_{s}$ valence band, and the alloy is a direct-gap semiconductor at the $L$ points. As $x$ is increased further, the gap increases until its maximum value of order $30 \mathrm{meV}$ at $x=0.18$. At that point, the valence band at $H$ crosses the $L_{s}$ valence band. For $x>0.22$, the $H$ band crosses the $L_{a}$ conduction band, and the alloy is again a semimetal.

Since the inversion transition between the $L_{s}$ and $L_{a}$ bands occurs in the semimetal phase adjacent to pure bismuth, it is clear that the semiconducting $\mathrm{Bi}_{1-x} \mathrm{Sb}_{x}$ alloy inherits its topological class from pure antimony and is thus a strong topological insulator. Of course, this conclusion is predicated on a "virtual-crystal approximation" in which the disorder due to the random mixture is ignored, so that inversion symmetry is preserved in the alloy. However, since this inherent disorder does not destroy the bulk energy gap, it is unlikely to change the topological class, which does not require inversion (or translation) symmetry. We thus conclude that intrinsic $\mathrm{Bi}_{1-x} \mathrm{Sb}_{x}$, despite its bulk energy gap, will have conducting surface states, which form a topological metal.

Semiconducting $\mathrm{Bi}_{1-x} \mathrm{Sb}_{x}$ alloys have been studied experimentally because of their thermoelectric properties, which make them desirable for applications as thermocouples. ${ }^{43,48-50}$ Transport studies have been carried out both on bulk samples ${ }^{43}$ and epitaxial thin films. ${ }^{49}$ For $T>50 \mathrm{~K}$, semiconducting behavior is observed, while at lower temperatures, the resistivity saturates at a value in the range $5-50 \mu \Omega m$. This observed residual resistivity is probably too small to be explained by surface states. It has been attributed to residual charged impurities, ${ }^{43}$ which act as shallow donors, making the alloy slightly $n$ type. In order to separate the surface properties from the bulk transport, it will be necessary either to improve the purity of the samples or perhaps use gating in a heterostructure to push the Fermi energy into the gap.

\section{B. Gray tin and mercury telluride}

Tin is a group-IV element, which in its $\alpha$ (or gray) phase has the inversion symmetric diamond structure. Unlike carbon, silicon, and germanium, though, it is a zero gap semiconductor, in which the ordering of the states at the conduction- and valence-band edge is inverted [see Fig. 6(a).$^{51,52}$ The Fermi energy lies in the middle of a fourfold
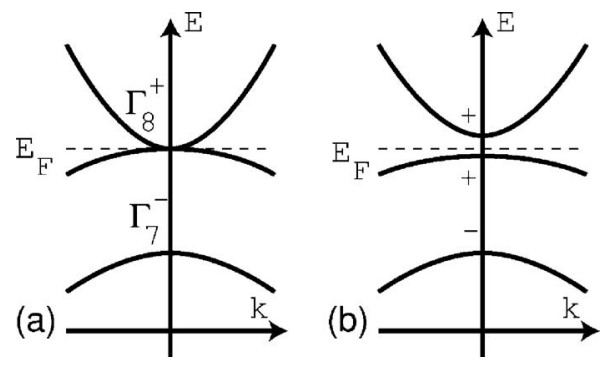

FIG. 6. (a) Band structure of $\alpha$-Sn near the $\Gamma$ point, which describes zero gap semiconductor due to the inverted $\Gamma_{8}^{+}$and $\Gamma_{7}^{-}$ bands. (b) In the presence of uniaxial strain, the degeneracy at $\Gamma$ is lifted, opening a gap in the spectrum. The parity eigenvalues remain unchanged.

degenerate set of states with $\Gamma_{8}^{+}$symmetry, which can be derived from $p$ states with total angular momentum $j=3 / 2$. The fourfold degeneracy at the $\Gamma_{8}^{+}$point is a consequence of the cubic symmetry of the diamond lattice. Applying uniaxial strain lifts this degeneracy into a pair of Kramers doublets and introduces an energy gap into the spectrum..$^{53}$ For pressures of order $3 \times 10^{9} \mathrm{dyn} / \mathrm{cm}^{2}$, the induced energy gap is of order $40 \mathrm{meV}$. We now argue that this insulating phase is, in fact, a strong topological insulator.

Table $\mathrm{V}$ shows the symmetry labels for unstrained $\alpha$-Sn associated with the four occupied valence bands at the eight time-reversal invariant momenta. ${ }^{54}$ Uniaxial strain lowers the symmetry, so the cubic symmetry labels no longer apply. However, since the strain does not violate inversion symmetry, the parity eigenvalues are unchanged. The only effect is to split the degeneracy of the $\Gamma_{8}^{+}$level into two sets of evenparity Kramers doublets [see Fig. 6(b)]. In Table V, $\Gamma_{8}^{+*}$ refers to the occupied doublet. Based on the parity eigenvalues, we conclude that strained gray tin is a strong topological insulator.

$\mathrm{HgTe}$ is a II-VI material with the zinc-blende structure $^{52,55}$ It is a zero gap semiconductor with an electronic structure closely related to gray tin. The Fermi energy is in the middle of the fourfold degenerate $\Gamma_{8}$ states, whose degeneracy follows from the cubic symmetry of the zincblende lattice. As in gray tin, uniaxial strain lifts this degeneracy and opens a gap at the Fermi energy.

Though HgTe lacks inversion symmetry, we now argue based on adiabatic continuity that the gap induced by uniaxial strain leads to a strong topological insulator. The electronic structure of II-VI materials can be understood by adding an inversion symmetry-breaking perturbation to a inversion symmetric group-IV crystal. ${ }^{52,56}$ Provided that this perturbation does not lead to any level crossings at the Fermi energy, we can conclude that the II-IV material is in the same

TABLE V. Symmetry labels for the four valence bands of gray tin at eight time-reversal invariant momenta, according to Ref. 54.

\begin{tabular}{lcc}
\hline \hline $1 \Gamma$ & $\Gamma_{6}^{+} \Gamma_{7}^{+} \Gamma_{7}^{-} \Gamma_{8}^{+*}$ & - \\
$3 X$ & $2 X_{5} 2 X_{5 v}$ & + \\
$4 L$ & $L_{6}^{-} L_{6}^{+} L_{6 v}^{-} L_{45}^{-}$ & - \\
& $Z_{2}$ Class & $(1 ; 000)$ \\
\hline \hline
\end{tabular}


TABLE VI. Symmetry labels for the five valence bands of tin telluride and lead telluride at eight time-reversal invariant momenta, according to Ref. 59.

\begin{tabular}{lcc}
\hline \hline & Tin Telluride & \\
$1 \Gamma$ & $\Gamma_{6}^{+} \Gamma_{6}^{+} \Gamma_{6}^{-} 2 \Gamma_{8}^{+}$ & - \\
$3 X$ & $X_{6}^{+} X_{6}^{+} X_{6}^{-} X_{6}^{-} X_{7}^{-}$ & - \\
$4 L$ & $L_{6}^{-} L_{6}^{+} L_{6}^{+} L_{45}^{+} L_{6}^{-}$ & + \\
& $Z_{2}$ class & $(0 ; 000)$ \\
& Lead telluride & \\
$1 \Gamma$ & $\Gamma_{6}^{+} \Gamma_{6}^{+} \Gamma_{6}^{-} 2 \Gamma_{8}^{+}$ & - \\
$3 X$ & $X_{6}^{+} X_{6}^{+} X_{6}^{-} X_{6}^{-} X_{7}^{-}$ & - \\
$4 L$ & $L_{6}^{-} L_{6}^{+} L_{6}^{+} L_{45}^{+} L_{6}^{+}$ & $(0 ; 000)$ \\
\hline \hline
\end{tabular}

topological class as the group-IV crystal. The band structures of gray tin and HgTe are very similar, and the cubic symmetry labels of the energy bands show how the bands evolve between the two. This allows us to conclude that strained $\alpha$-Sn and $\mathrm{HgTe}$ will be in the same topological class, which is that of the strong topological insulator.

In Ref. 25, Murakami et al. introduced a four band tightbinding model based on $p_{3 / 2}$ atomic levels on a fcc lattice to describe strained $\alpha$-Sn and HgTe. As argued in Ref. 10, this model predicts that these materials are simple insulators in the $0 ;(000)$ class. This can be understood by noting that since the model includes only $p_{3 / 2}$ atomic levels, the parity eigenvalues in Eq. (1.1) are all $\xi_{i}=-1$. This contradicts the known band structure of these materials, as displayed in Table V. This model correctly describes the electronic states near the $\Gamma$ point, but it gets the global topology of the bands wrong. To capture the global topology, a tight-binding model of these materials must include both $s$ and $p$ levels. The more recent theory ${ }^{40}$ of the $2 \mathrm{D}$ quantum spin-Hall effect in $\mathrm{HgTe} / \mathrm{CdTe}$ quantum wells discussed in Sec. IV D correctly incorporates $s$ and $p$ levels.

\section{Lead-tin telluride}

$\mathrm{PbTe}$ and SnTe are narrow gap IV-VI semiconductors with the rocksalt structure. ${ }^{57}$ The band gap in these materials is direct and occurs at the four equivalent $L$ points in the fcc Brillouin zone. PbTe has an inverted band structure relative to $\mathrm{SnTe}$, in which the relative ordering of the $L_{6}^{+}$and $L_{6}^{-}$ bands at the conduction- and valence-band edges are switched. Nonetheless, both of these materials are conventional insulators. In Table VI, we display the symmetry labels at the eight time-reversal invariant points $(\Gamma$, three equivalent $X$ points, and four equivalent $L$ points). ${ }^{59}$ Since the inversion occurs at an even number of points in the Brillouin zone, both materials belong to the conventional insulator topological class.

$\mathrm{PbTe}$ and $\mathrm{SnTe}$ form an alloy $\mathrm{Pb}_{1-x} \mathrm{Sn}_{x} \mathrm{Te}$. At $x \sim 0.4$, there is an inversion transition where the band gap at the four $L$ points vanishes, giving rise to three-dimensional Dirac points. ${ }^{57,58}$ The phases on either side of this transition are only distinct if inversion symmetry is present. Thus, disorder,

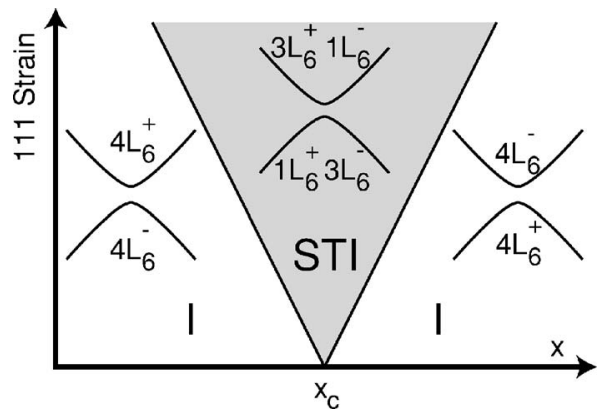

FIG. 7. Schematic uniaxial strain-composition phase diagram for $\mathrm{Pb}_{1-x} \mathrm{Sn}_{x} \mathrm{Te}$. Away from the inversion transition at $x \sim 0.4$, the material is a conventional insulator (I). Near the transition, it is a strong topological insulator (STI).

which is inevitably present in the alloy, blurs the transition. However, uniaxial strain oriented along the 111 direction will distinguish one of the $L$ points (call it $T$ now) from the other three $L$ points. It follows that the inversion transitions at the $L$ and $T$ points will occur at different values of $x$. Thus, there will be an intermediate phase in which $L$ is inverted, but $T$ is not (or vice versa). From Eqs. (1.1) and (1.2), this intermediate phase will be a strong topological insulator with surface states forming a topological metal. Note that this direction depends on the orientation of the uniaxial strain. For instance, strain along the 100 direction will distinguish two $L$ points from the other two and will not lead to an intermediate topological phase. In Fig. 7, we show a schematic phase diagram as a function of $x$ and 111 strain.

The band inversion between SnTe and PbTe has been discussed by a number of authors previously. Volkov and Pankratov $^{60}$ argued that PbTe and SnTe can be described by a low-energy field theory consisting of three-dimensional Dirac fermions with masses of opposite signs. They concluded that a planar interface between PbTe and SnTe will exhibit interface states described by a two-dimensional massless Dirac equation. The appearance of such domain-wall fermions is similar to the appearance of midgap states in a one-dimensional Peierls insulator at a soliton. ${ }^{34} \mathrm{~A}$ related proposal was made by Fradkin and co-workers, ${ }^{36,37}$ who considered a domain wall in PbTe in which on one side the $\mathrm{Pb}$ and Te atoms are interchanged. This was also modeled as 3D Dirac fermions with a mass which changes sign at the interface.

The domain-wall fermions which appear in these theories are similar to the states we predict at the surface of a strong topological insulator. Indeed, if one views the vacuum as a band insulator with a large gap, then the surface can be viewed as an interface between a band insulator and a topological insulator, which can be described as an inversion transition, where there is a change in the sign of the mass of a 3D Dirac fermion. However, there is an important difference between the proposals discussed above and the surface states of the topological insulator: the strong topologicalinsulator-band-insulator interface involves a sign change in an odd number of Dirac points, while the interface models above involve four Dirac points. Having an odd number is crucial for the topological stability of the surface states. 


\section{Other materials}

The materials we have proposed above should not be considered to be an exhaustive list. In general, it is necessary to consider insulators composed of heavy elements. Another candidate for a topological insulating phase is $\mathrm{Bi}_{2} \mathrm{Te}_{3}$, which, like $\mathrm{Bi}_{1-x} \mathrm{Sb}_{x}$, is known for its thermoelectric properties. ${ }^{61}$ This material is also a narrow gap semiconductor, with an energy gap of order $0.13 \mathrm{eV}$. Though the crystal structure of this material is inversion symmetric, we have been unable to locate band theory calculations which display the parity eigenvalues.

Another possible candidate is the zinc-blende semiconductor $\beta-\mathrm{HgS}$. The electronic structure of this material has been a subject of some controversy. According to Delin, ${ }^{62}$ it is a semiconductor which has an unusual band ordering, with the $\Gamma_{6}$ and $\Gamma_{8}$ levels in the valence band and the $\Gamma_{7}$ level in the conduction band. If this is the case, we expect the material to be a strong topological insulator. However, this conclusion has been challenged by Moon and Wei, ${ }^{63}$ who find a more conventional band ordering with the $\Gamma_{6}$ level in the conduction band and the $\Gamma_{7}$ and $\Gamma_{8}$ levels in the valence band, leading to a conventional insulator.

\section{EXPERIMENTAL IMPLICATIONS}

We now briefly consider possible experimental probes of topological insulators. We will focus here on the threedimensional strong topological insulator phase, for which we suggested several materials in the previous section.

The most direct probe of the topological insulators is transport. Since there is a bulk excitation gap, transport in intrinsic samples at very low temperature will be dominated by the surfaces, which can be probed by the geometry dependence of the conductance. For example, for a wire geometry, the conductance will be proportional to the circumference of the wire, rather than the area.

In addition, since the topological metal phase is in the symplectic universality class the conductivity is expected to increase logarithmically at low temperature: $\sigma(T)$ $\propto \log \left[L_{\text {in }}(T) / \ell\right],{ }^{64}$ where $L_{\text {in }}$ is the inelastic scattering length and $\ell$ is the mean free path.

An interesting prediction for the surface states is that due the $\pi$ Berry's phase associated with the surface Fermi arc, the surface quantum Hall effect in a perpendicular magnetic field should be quantized in half odd integers, $\sigma_{x y}=(n$ $+1 / 2) e^{2} / h$. As discussed in Sec. II C 1 , this is difficult to measure directly without separately measuring the currents flowing on the top and bottom surfaces of the sample. However, if the parallel combination of the two surfaces could be measured, the resulting Hall effect would be quantized in odd multiples of $e^{2} / h$. This is similar to the quantum Hall effect in graphene, ${ }^{65,66}$ which is quantized in odd multiples of $2 e^{2} / h$. The difference is due to the fact that graphene has four Dirac points, including spin.

A practical difficulty with transport measurements is that it is necessary to distinguish surface currents from bulk currents. Since bulk currents scale with the sample width $W$, even though there is a bulk energy gap $E_{g}$, the temperature must be low: $T \ll E_{g} / \log (W / a)$, where $a$ is the lattice constant. Moreover, since the materials we have suggested have rather small energy gaps, samples with high purity will be required to reach the intrinsic limit. As discussed in Sec. IV $\mathrm{A}$, the low-temperature behavior of $\mathrm{Bi}_{1-x} \mathrm{Sb}_{x}$ is dominated by a low concentration of charged impurities, which form an impurity band. ${ }^{43}$ This is a ubiquitous problem for narrow gap semiconductors due to their low effective mass and high dielectric constant. Clearly, it would be desirable to have a transport geometry which probes the surface states while controlling the position of the bulk Fermi energy. Perhaps, this may be possible in a clever heterostructure geometry, where the bulk Fermi energy can be adjusted with a gate.

An alternative probe of the surface states would be to map the surface Fermi arc using angle-resolved photoemission. Such measurements could establish that the surface Fermi arc encloses an odd number of time-reversal invariant momenta in the strong topological insulator phase. Detailed angle-resolved photoemission spectroscopy studies have been carried out on the surfaces of bismuth ${ }^{67-69}$ and antimony. ${ }^{70}$ However, the presence of the bulk Fermi surface complicates the analysis of these materials. It would be interesting to see how the results are modified in the semiconducting $\mathrm{Bi}_{1-x} \mathrm{Sb}_{x}$ alloy.

Finally, since the surface states are spin filtered, electrical currents flowing on the surface will be associated with spin accumulation, leading to a spin-Hall effect. In GaAs, spin accumulation on a surface has been measured. ${ }^{71,72}$ The narrow energy gaps in our proposed materials make detection of the spin accumulation more difficult. Perhaps, a heterostructure geometry could make this possible.

\section{CONCLUSION}

In this paper, we discussed topological insulator phases in two and three dimensions. We discussed in detail how the $Z_{2}$ topological invariants characterizing these phases influence the surface-state spectrum. In particular, the quantum spinHall phase in two dimensions and the strong topological insulator in three dimensions have robust conducting surface states, which we have characterized as a topological metal. We showed that the $Z_{2}$ invariants can be determined easily in parity invariant crystals from the knowledge of the parity eigenvalues for states at the time-reversal invariant points in the Brillouin zone. Using this method, we deduced that the semiconducting alloy $\mathrm{Bi}_{1-x} \mathrm{Sb}_{x}$ is a strong topological insulator, as are $\alpha$-Sn and HgTe in the presence of uniaxial strain.

There remain a number of further issues which need to be understood better. High among them are the effects of disorder and interactions. These are important both for the topological metal surface states as well as for the bulk topological phases. Numerical work by Onoda et al. ${ }^{73}$ has suggested that the transition between the conventional insulator and the quantum spin-Hall phase in two dimensions belongs to a new universality class. It will be of interest to understand this transition better, along with the related transition between the topological insulator and the Anderson insulator, which presumably occurs when disorder is increased beyond a critical value. 
Finally, it would be desirable to develop a field theory for the topological insulating phases analogous to the ChernSimons theory of the quantum Hall effect. Perhaps, this may lead to analogs of the fractional quantum Hall effect for the topological insulators.

\section{ACKNOWLEDGMENTS}

It is a pleasure to thank Eduardo Fradkin and Gene Mele for helpful discussions. This work was supported by the NSF Grant No. DMR-0605066 and by ACS PRF Grant No. 44776-AC10.
${ }^{1}$ W. Kohn, Phys. Rev. 133, A171 (1964).

${ }^{2}$ X. G. Wen, Phys. Rev. B 44, 2664 (1991).

${ }^{3}$ E. Demler, C. Nayak, H. Y. Kee, Y. B. Kim, and T. Senthil, Phys. Rev. B 65, 155103 (2002), and references therein.

${ }^{4}$ F. D. M. Haldane, Phys. Rev. Lett. 61, 2015 (1988).

${ }^{5}$ D. J. Thouless, M. Kohmoto, M. P. Nightingale, and M. den Nijs, Phys. Rev. Lett. 49, 405 (1982).

${ }^{6}$ M. Kohmoto, B. I. Halperin, and Y.-S. Wu, Phys. Rev. B 45, 13488 (1992).

${ }^{7}$ B. I. Halperin, Phys. Rev. B 25, 2185 (1982).

${ }^{8}$ Y. Hatsugai, Phys. Rev. Lett. 71, 3697 (1993).

${ }^{9}$ C. L. Kane and E. J. Mele, Phys. Rev. Lett. 95, 226801 (2005).

${ }^{10}$ C. L. Kane and E. J. Mele, Phys. Rev. Lett. 95, 146802 (2005).

${ }^{11}$ B. A. Bernevig and S. C. Zhang, Phys. Rev. Lett. 96, 106802 (2006)

${ }^{12}$ R. Roy, arXiv:cond-mat/0604211 (unpublished).

${ }^{13}$ J. E. Moore and L. Balents, Phys. Rev. B 75, 121306(R) (2007).

${ }^{14}$ R. Roy, arXiv:cond-mat/0607531(unpublished).

${ }^{15}$ L. Fu, C. L. Kane, and E. J. Mele, Phys. Rev. Lett. 98, 106803 (2007).

${ }^{16}$ H. Suzuura and T. Ando, Phys. Rev. Lett. 89, 266603 (2002).

${ }^{17}$ F. D. M. Haldane, Phys. Rev. Lett. 93, 206602 (2004).

${ }^{18}$ S. Hikami, A. I. Larkin, and Y. Nagaoka, Prog. Theor. Phys. 63, 707 (1980).

${ }^{19}$ H. Nielssen and N. Ninomiya, Phys. Lett. 130B, 389 (1983).

${ }^{20}$ D. N. Sheng, Z. Y. Weng, L. Sheng, and F. D. M. Haldane, Phys. Rev. Lett. 97, 036808 (2006).

${ }^{21}$ T. Fukui and Y. Hatsugai, arXiv:cond-mat/0607484 (unpublished).

${ }^{22}$ L. Fu and C. L. Kane, Phys. Rev. B 74, 195312 (2006).

${ }^{23}$ M. Kohmoto, Ann. Phys. (N.Y.) 160, 343 (1985).

${ }^{24}$ S. Murakami, Phys. Rev. Lett. 97, 236805 (2006).

${ }^{25}$ S. Murakami, N. Nagaosa, and S. C. Zhang, Phys. Rev. Lett. 93, 156804 (2004).

${ }^{26}$ R. B. Laughlin, Phys. Rev. B 23, R5632 (1981).

${ }^{27}$ E. I. Blount, Solid State Phys. 13, 305 (1962).

${ }^{28}$ J. Zak, Phys. Rev. Lett. 62, 2747 (1989).

${ }^{29}$ R. D. King-Smith and D. Vanderbilt, Phys. Rev. B 47, 1651 (1993).

${ }^{30}$ R. Resta, Rev. Mod. Phys. 66, 899 (1994).

${ }^{31}$ C. Wu, B. A. Bernevig, and S. C. Zhang, Phys. Rev. Lett. 96, 106401 (2006).

${ }^{32}$ C. Xu and J. E. Moore, Phys. Rev. B 73, 045322 (2006).

${ }^{33}$ D. B. Kaplan, Phys. Lett. B 288, 342 (1992).

${ }^{34}$ W. P. Su, J. R. Schrieffer, and A. J. Heeger, Phys. Rev. Lett. 42, 1698 (1979).

${ }^{35}$ R. Jackiw, Phys. Rev. D 29, 2375 (1984).

${ }^{36}$ E. Fradkin, E. Dagotto, and D. Boyanovsky, Phys. Rev. Lett. 57, 2967 (1986).

${ }^{37}$ D. Boyanovsky, E. Dagotto, and E. Fradkin, Nucl. Phys. B 285,
240 (1987)

${ }^{38}$ G. W. Semenoff, Phys. Rev. Lett. 53, 2449 (1984).

${ }^{39}$ Q. Niu, D. J. Thouless, and Y. S. Wu, Phys. Rev. B 31, 3372 (1985).

${ }^{40}$ A. Bernevig, T. Hughes, and S. C. Zhang, Science 314, 1757 (2006).

${ }^{41}$ S. Murakami, N. Nagaosa, and S. C. Zhang, Science 301, 1348 (2003); Phys. Rev. B 69, 235206 (2004).

${ }^{42}$ L. S. Lerner, K. F. Cuff, and L. R. Williams, Rev. Mod. Phys. 40, 770 (1968).

${ }^{43}$ B. Lenoir, M. Cassart, J.-P. Michenaud, H. Scherrer, and S. Scherrer, J. Phys. Chem. Solids 57, 89 (1996); B. Lenoir, A. Dauscher, X. Devaux, R. Martin-Lopez, Yu. I. Ravich, H. Scherrer, and S. Scherrer, Proceedings of 15th International Conference on Thermoelectrics, 1996 (unpublished), pp. 1-13.

${ }^{44}$ S. Golin, Phys. Rev. 166, 643 (1968).

${ }^{45}$ Y. Liu and R. E. Allen, Phys. Rev. B 52, 1566 (1995).

${ }^{46}$ L. M. Falicov and S. Golin, Phys. Rev. 137, A871 (1965).

${ }^{47}$ S. Golin, Phys. Rev. 176, 830 (1968).

${ }^{48}$ D. T. Morelli, D. L. Partin, and J. Heremans, Semicond. Sci. Technol. 5, S257 (1990).

${ }^{49}$ S. Cho, A. DiVenere, G. K. Wong, J. B. Ketterson, and J. R. Meyer, Phys. Rev. B 59, 10691 (1999).

${ }^{50}$ Y. Lin et al., Appl. Phys. Lett. 79, 677 (2001).

${ }^{51}$ S. Groves and W. Paul, Phys. Rev. Lett. 11, 194 (1963).

${ }^{52}$ I. A. Tsidikovski, Electron Spectrum of Gapless Semiconductors, Springer Series in Solid State Sciences Vol. 116 (SpringerVerlag, Berlin, 1997).

${ }^{53}$ B. J. Roman and A. W. Ewald, Phys. Rev. B 5, 3914 (1972).

${ }^{54}$ F. H. Pollak, M. Cardona, C. W. Higginbotham, F. Herman, and J. P. Van Dyke, Phys. Rev. B 2, 352 (1970).

${ }^{55}$ R. Dornhaus and G. Nimtz, Narrow Gap Semiconductors, Springer Tracts in Modern Physics Vol. 98 (Springer-Verlag, Berlin, 1983).

${ }^{56}$ F. Herman, Electronics 1, 103 (1955).

${ }^{57}$ G. Nimtz and B. Schicht, Narrow Gap Semiconductors, Springer Tracts in Modern Physics Vol. 98 (Springer-Verlag, Berlin, 1983).

${ }^{58}$ J. Dimmock, I. Melngailis, and A. J. Strauss, Phys. Rev. Lett. 16, 1193 (1966).

${ }^{59}$ Y. W. Tung and M. L. Cohen, Phys. Rev. 180, 823 (1969).

${ }^{60}$ B. A. Volkov and O. A. Pankratov, Pis'ma Zh. Eksp. Teor. Fiz. 42, 145 (1985) [JETP Lett. 42, 178 (1985)].

${ }^{61}$ S. K. Mishra, S. Satpathy, and O. Jepsen, J. Phys.: Condens. Matter 9, 461 (1997).

${ }^{62}$ A. Delin, Phys. Rev. B 65, 153205 (2002).

${ }^{63}$ C. Y. Moon and S. H. Wei, Phys. Rev. B 74, 045205 (2006).

${ }^{64}$ T. Senthil and M. P. A. Fisher, Phys. Rev. B 61, 9690 (2000).

${ }^{65}$ K. S. Noveselov, A. K. Geim, S. V. Morozov, D. Jiang, M. I. Katsnelson, I. V. Grigorieva, S. V. Dubonos, and A. A. Firsov, 
Nature (London) 438, 197 (2005).

${ }^{66}$ Y. Zhang, Y.-W. Tan, H. L. Stormer, and P. Kim, Nature (London) 438, 201 (2005).

${ }^{67}$ C. R. Ast and H. Höchst, Phys. Rev. Lett. 87, 177602 (2001).

${ }^{68}$ J. E. Gayone, S. V. Hoffmann, Z. Li, and P. Hofmann, Phys. Rev. Lett. 91, 127601 (2003).

${ }^{69}$ P. Hofmann, J. E. Gayone, G. Bihlmayer, Y. M. Koroteev, and E. V. Chulkov, Phys. Rev. B 71, 195413 (2005).
${ }^{70}$ K. Sugawara, T. Sato, S. Souma, T. Takahashi, M. Arai, and T. Sasaki, Phys. Rev. Lett. 96, 046411 (2006).

${ }^{71}$ Y. K. Kato, R. C. Myers, A. C. Gossard, and D. D. Awschalom, Science 306, 1910 (2004).

${ }^{72}$ J. Wunderlich, B. Kaestner, J. Sinova, and T. Jungwirth, Phys. Rev. Lett. 94, 047204 (2005).

${ }^{73}$ M. Onoda, Y. Avishai, and N. Nagaosa, Phys. Rev. Lett. 98, 076802 (2007). 